\title{
Le climat de Laval au début de la Renaissance
}

Essai de géographie historique

Laval's climate in the early Renaissance. An essay in historical geography

Jean-Pierre Marchand, Valérie Bonnardot et Olivier Planchon

\section{(2) OpenEdition \\ Journals}

Édition électronique

URL : http://journals.openedition.org/abpo/2973

DOI : $10.4000 /$ abpo.2973

ISBN : 978-2-7535-4130-6

ISSN : 2108-6443

Éditeur

Presses universitaires de Rennes

Édition imprimée

Date de publication : 15 avril 2015

Pagination : 103-133

ISBN : 978-2-7535-4128-3

ISSN : 0399-0826

Référence électronique

Jean-Pierre Marchand, Valérie Bonnardot et Olivier Planchon, « Le climat de Laval au début de la Renaissance », Annales de Bretagne et des Pays de l'Ouest [En ligne], 122-1 | 2015, mis en ligne le 15 avril 2017, consulté le 19 avril 2019. URL : http://journals.openedition.org/abpo/2973; DOI : 10.4000/ abpo. 2973 


\title{
Le climat de Laval au début de la Renaissance
}

\author{
Essai de géographie historique
}

\author{
Jean-Pierre Marchand, Valérie BonnARdot, Olivier Planchon \\ Professeur émérite Université Rennes2, UMR 6554 LETG-COSTEL \\ Maître de conférences Université Rennes2, UMR 6554 LETG-COSTEL \\ Chargé de recherches CNRS, UMR 6554 LETG-COSTEL
}

" Chaque année, moissons et vendanges posent aux hommes désarmés une quête angoissante dont la réponse, de juillet à août, dépend du soleil et de la pluie des mois précédents " (François Lebrun).

Les " Annales et chroniques du païs de Laval depuis l'an 1480 [AS] jusqu'à l'année 1537 [AS] ${ }^{1}$ ", que le notaire du comté de Laval, Guillaume Le Doyen, a rédigées, en vers de médiocre qualité, fournissent des renseignements forts précieux sur la vie lavalloise entre 1481 (NS) et 1538 (NS). Ce manuscrit est conservé aux archives départementales de la Mayenne et a été édité au $\mathrm{XIX}^{\mathrm{e}}$ siècle par M. Godbert ${ }^{2}$. Le Doyen indique entre autres les épidémies, les malheurs des guerres; à ce titre, il a été utilisé par H. Neveux et J. Céard dans l'ouvrage dirigé par Jean Delumeau et Yves Lequin, Les malheurs des temps ${ }^{3}$. Il a été souvent cité par les érudits mayennais du XIX ${ }^{\mathrm{e}}$ siècle et les historiens du XXe siècle $^{4}$; la seule étude globale et récente est celle de Wan Chun Sung ${ }^{5}$.

1. AS = Ancien Style $; \mathrm{NS}=$ Nouveau Style. Dans l'ancien style, le début de l'année est à Pâques; dans le nouveau style, il est au premier janvier. À l'époque où écrit Le Doyen, le nouveau style ne s'est pas encore définitivement imposé partout, il ne sera généralisé que par l'ordonnance de Charles IX de 1564, cf. note 7 infra (NDLR).

2. Ouvrage disponible en ligne sur Gallica.

3. NEveuX, Hugues, CEARD, Jean, « Rémission des fléaux et attente des merveilles ", dans DeluMEAU, Jean et LEQuin, Yves, Les malheurs des temps. Histoire des fléaux et des calamités en France, Paris, Larousse, 1987, p. 244-245.

4. Angot, Alphonse, Dictionnaire historique, topographique et biographique de la Mayenne, 4 tomes, Laval, imp. Goupil, 1900-1910; PIchoт, Daniel, " Le Moyen Âge ", dans SALBERT, Jacques (dir.), La Mayenne des origines à nos jours, Saint-Jean-d'Angély, Bordessoules, 1984, p. 97-172; SALBERT, Jacques, « La Mayenne des temps modernes (XVIXVIII siècles) ", dans : SALBERT, Jacques (dir.), La Mayenne des origines..., op. cit., p. 173-298.

5. Sung, Wan Chung, La Chronique de Guillaume Le Doyen, master d'histoire médiévale, directeur Daniel Pichot, Université Rennes 2, inédit, 2007. 
À la lecture du texte, il apparaît que, pour les années de la fin du XV siècle, le notaire a rédigé sa chronique tardivement, après 1505 selon $\mathrm{M}$. Le Mené, peut-être en s'appuyant sur des notes écrites au fil du temps ${ }^{6}$; en effet, la structuration du texte change alors et devient plus ordonnée. Pour les vingt premières années de la chronique de Le Doyen, soit la fin du Xve siècle, le désordre de la rédaction est si prononcé que les informations sur les hivers ne peuvent être datées avec certitude (la marge d'erreur est d'un an) et la chronologie n'est pas sûre, à l'exception de quelques événements marquants comme le grand hiver de 1481 (NS). À partir des années 1501-1505 (NS), la description de l'hiver est placée à la fin du développement sur chaque année; il devient alors beaucoup plus facile de déterminer de quel hiver il parle, c'est bien l'hiver qui clôt l'année dans le calendrier ancien style (AS) ${ }^{7}$.

Dans sa chronique, G. Le Doyen fait une place importante au climat; il décrit chaque année le temps des hivers, la qualité des récoltes de blé et celle des vendanges, comme le montre le début du récit de l'année 1518, assez caractéristique d'un aléa hivernal et d'une vulnérabilité de printemps et d'été. Lorsque les informations météorologiques font défaut, ce qui est fréquent, les caractéristiques des saisons ont alors été déduites des appréciations sur les récoltes.

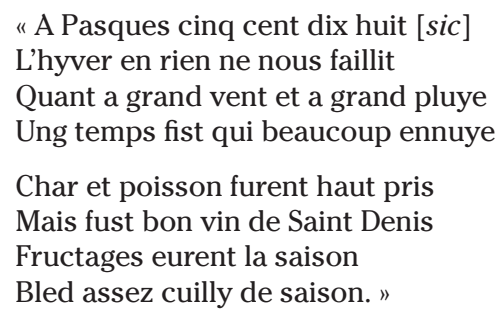

Il nous donne ainsi une chronique régulière permettant de reconstituer l'évolution du climat sur cinquante-sept ans. Or cet aspect du travail de Le Doyen est ignoré chez Emmanuel Le Roy Ladurie ${ }^{8}$ et n'a pas été étudié systématiquement dans une optique d'évolution du climat par les historiens, sauf par Michel Le Mené qui l'utilise dans sa thèse ${ }^{9}$ comme cadre de la vie rurale en Anjou au Moyen Âge et par Alain Croix comme facteur de la démographie de la Bretagne au début de l'époque moderne. Hugues Neveux et Jean Céard citent sa description du terrible hiver $1481^{10}$.

6. Le Mené, Michel, Les campagnes angevines à la fin du Moyen Âge, Nantes, Cid éditions, 1982, p. 61, n.18.

7. Depuis l'édit de Roussillon (1564) ayant fixé le début de l'année au $1^{\mathrm{e}}$ janvier, les trois mois d'hiver (janvier, février, mars) constituent le premier trimestre de l'année (NS). Pour éviter toute confusion, les dates concernant les hivers seront données dans le développement en nouveau style, soit l'année indiquée par Le Doyen + 1. La chronique commence donc à l'hiver 1481 (NS) et finit avec celui de 1538 (NS).

8. Le Roy Ladurie, Emmanuel, Histoire du climat depuis l'an mil, Tome 1, Paris, Flammarion, 1986 (1 ${ }^{\mathrm{e}}$ édition 1967). Id., Histoire humaine et comparée du climat, Tome 1, Paris, Fayard, 2004.

9. Le MENÉ, Michel, Les campagnes angevines..., op. cit., Nantes, Cid éditions, 1982.

10. NeveuX, Hugues, CÉARD, Jean, « Rémission des fléaux... », op. cit., p. 244. 
Rares sont les événements datés : ils ne concernent que 21 années sur les 57 de la chronique, pour 30 occurrences. Le Doyen n'a pas tenu quotidiennement un journal décrivant le temps au jour le jour, comme le fera au milieu du XVI ${ }^{\mathrm{e}}$ siècle le sieur de Gouberville ${ }^{11}$. Il note les neuf crues ayant touché les bas quartiers de Laval sur la rive gauche, avec des descriptions suffisamment précises pour que l'on puisse délimiter les débordements des crues décennales et trentennales ${ }^{12}$.

La mémoire de Le Doyen est très sélective pour ce qui est des événements datés avec précision. Sur les 31 cas recensés, la datation se fait d'après le calendrier religieux comme il est habituel : " grêle à la Fête Dieu " 1501, " gelées à la Pentecôte " 1502, " beau vendredi saint " 1521, orage à la SaintJean 1523. Il note le beau temps du 4 mai 1487 lors de l'arrivée du roi Charles VIII, et du 7 août 1487 lors de l'entrée de Guy XV à Laval, la pluie et les vents forts lors de la visite d'Antoinette du Lude le 21 septembre 1526. Il a fallu des occasions rares ou des fêtes carillonnées pour que Le Doyen les signale, ce qui suppose une sous-estimation vraisemblable de tels types d'accidents. L'expression, encore à l'honneur dans la presse de nos jours, "de mémoire d'homme " revient à plusieurs reprises sous sa plume (en 1531 par exemple). Il signale également des événements survenus à proximité de Laval qui relèvent plus de l'anecdote que de la compilation climatique : le 18 octobre 1512 , " un vent brûlant comme un lutin tomba à terre le clocher d'Ahuillé sans rien blesser qu'un pauvre vicaire ". Il a connaissance des crues de la Loire à Orléans et Saumur en 1527. Comme souvent, ce sont des événements catastrophiques, des exemples de mauvais temps ou d'aléas climatiques ayant des répercussions sur l'approvisionnement des villes, sur les famines, qui sont pris en compte. On retrouve cette approche à Paris aussi bien dans le livre de raison de Nicolas Versoris ${ }^{13}$ que dans le Journal d'un bourgeois de Paris sous le règne de François $1^{\text {er14 }}$, œuvres contemporaines de celle de Le Doyen. On retrouvera cette tendance aux " vents mauvais " pour Laval dans les chroniques de Duchemin du Tertre ${ }^{15}$ et de Guittet de La Houllerie au XVIII ${ }^{\mathrm{e}}$ siècle $^{16}$.

Si Le Doyen fournit des données permettant une reconstitution des types de temps saisonniers, il donne peu de renseignements sur les causes

11. FoIsIL, Madeleine, Le Sire de Gouberville : un gentilhomme normand au XVI ${ }^{e}$ siècle, Paris, Flammarion, 1986.

12. MARChAND, Jean-Pierre, BonNARDOT, Valérie, " Les territoires du risque à Laval et le climat (1480 1537)", Actes du XV colloque AIC, Grenoble, 2012, p. 499-503.

13. Livre de raison de Nicolas Versoris, avocat au parlement de Paris (1519-1530), publié par G. FAGniEz, Société de l'histoire de Paris, 1885. Ouvrage disponible en ligne sur "Université d'Ottawa ".

14. Journal d'un bourgeois de Paris sous le règne de François Ir (1515-1536), publié par Lalanne, Ludovic, Paris, Jules Renard édit. et Librairie de la Société d'histoire de France, 1854. Ouvrage disponible en ligne sur Gallica.

15. AnToIne, Annie, Les comptes ordinaires de Pierre Duchemin du Tertre, Laval, Société d'archéologie et d'histoire de la Mayenne, 1998.

16. Mémoire chronologique de Maucourt de Bourjolly sur la ville de Laval suivi de la chronique de Guittet de la Houllerie, texte établi par Jules FizELIER, publié avec de nouvelles recherches par BERTRAND DE BROUSSILLON, 1886. Ouvrage disponible en ligne sur Gallica. 
des accidents météorologiques constatés. En 1501, «tombèrent des grêlons qu'on disait procéder de neige qui par avant ou pays d'amont [direction N-NE] avait été entre les monts " (les collines au nord de Laval?) et en 1509, " la rousée, sans contredit du ciel d'amont si descendit ". Il invoque à la Saint-Martin 1534 " le vent de galesnes qui gela toute la contrée ". Et il se félicite en 1514 de l'existence du bocage ${ }^{17}$ : «Si n'étaient taillis et bocages, nous aurions grands froids aux naiges, toute l'année pour les grandes pluies et aussi pour les grands vents de l'année présente. "

De même, les recours classiques aux cérémonies religieuses ${ }^{18}$ et aux processions pour faire cesser la pluie ou le froid reviennent régulièrement. En 1508, "l'église par procession/faisait et aussi oraisons/tellement l'aout fût moult beau " et en 1509, " la rosée fût bien venue à Pâques car le peuple se disposa à recevoir Dieu ». En 1509, il note également : "Pour bien chanté dieu louanges/nous eûmes très bonnes vendanges. "

À partir de la chronique de Guillaume Le Doyen, le but de cet article est triple:

- estimer la variabilité interannuelle du climat à Laval sur cette période de près de 56 ans, soit l'équivalent de deux normales climatiques;

- se demander si la rémission du petit âge glaciaire (PAG) due à une augmentation des températures décrite par E. Le Roy Ladurie pour la période 1480-1540 peut être observée dans l'Ouest de la France;

- aborder la thématique du risque d'origine climatique en analysant la fréquence et l'importance des crues à Laval telles que Le Doyen les a vécues.

Pour le géographe, le climat est une ressource naturelle dont on doit étudier le gisement ${ }^{19}$ : l'étude de la variabilité interannuelle en découle. Mais en tant qu'élément de l'infrastructure économique régionale, il nous permet, comme cela été fait pour la grande famine irlandaise ${ }^{20}$, d'aborder les relations "nature/société " à travers les conséquences des risques naturels comme les inondations. Outre ces deux objectifs, C.-P. Péguy et J.-P. Marchand en ont proposé une troisième, qui est l'étude du climat vécu. C'est cet aspect de la recherche climatique qui va être au cœur de la reconstitution du climat lavallois à partir de la chronique de G. Le Doyen.

Elle répond au souhait d'Hervé Le Treut qui, en 2011, écrivait que « des études concernant la vulnérabilité des différents territoires à l'évolution des paramètres climatiques sont nécessaires, car ce sont les seules études qui

17. Antorne, Annie, Le paysage de l'historien. Archéologie des bocages de l'ouest de la France à l'époque moderne, Rennes, PUR, 2002.

18. MinoIs, Georges, "Le climat, le dîmes et les prix trégorrois à travers le culte de saint Yves (XVII $-\mathrm{e}$-XIII ${ }^{\mathrm{e}}$ siècles)", Annales de Bretagne et des pays de l'Ouest, T. 88, ${ }^{\circ} 1,1981$, p. $87-108$.

19. PÉGuY, Charles Pierre et MARChAND, Jean-Pierre, "Climatologie et culture ", L'espace géographique, 3, 1982, p. 185-195.

20. MARChAND, Jean-Pierre, Contraintes climatiques et espace géographique, le cas Irlandais, Caen, Paradigme, 1985. 
permettront de placer les changements à venir, anthropiques ou naturels, dans un contexte interdisciplinaire large, permettant d'associer des facteurs socio-économiques ou écologiques à l'étude physique du climat ${ }^{21}$ ".

\section{Méthodologie}

Les propositions classiques d'E. Le Roy Ladurie ${ }^{22}$ pour la reconstitution des climats passés prennent en compte les dates de vendanges, le prix du blé, le gel des canaux et les avancées des glaciers. Quant aux mesures, la longue série des températures de Paris reconstituée par D. Rousseau ${ }^{23}$ ne commence qu'en $1676 .$.

\section{La qualité des sources}

$\mathrm{Au} \mathrm{XVI}^{\mathrm{e}}$ siècle, dans le Bas Maine, ces données sont absentes ou peu exploitables. Les glaciers, évidemment sont absents! La Mayenne n'est pas encore canalisée et le gel est trop aléatoire dans le temps en climat océanique pour que les chroniques en tiennent compte. Les données des vendanges des abbayes ne sont pas disponibles car ces dernières ne produisent plus de vin et préfèrent en importer depuis l'Anjou ${ }^{24}$. Pour ces raisons, le climat de l'Ouest de la France est peu connu en comparaison de celui de l'Est ou du Bassin Parisien et ce, jusqu'au début du XvIII ${ }^{\mathrm{e}}$ siècle. Surtout, les chroniques continues sur de longues périodes sont rares : M. Le Mené pour les décennies entre 1470 et $1540^{25}$ et A. Croix sur la période 1451$1670^{26}$ recoupent diverses sources dispersées sur la Bretagne, le Maine et l'Anjou. Le tableau dressé par A. Croix montre bien les discontinuités temporelles sur ces 220 années. Dans ces deux thèses, la seule continuité temporelle digne de ce nom est celle fournie entre 1480 et 1537 par Guillaume Le Doyen, d'où son intérêt, non seulement pour Laval mais aussi pour tout l'Ouest de la France. "Le puzzle documentaire " signalé à juste titre par E. Garnier ${ }^{27}$ auquel est confronté tout historien du climat se réduit ici à

21. LE Treut, Hervé, «Modèles climatiques, certitudes, incertitudes et impacts locaux ”, Actes du XXII colloque AIC, Rennes, 2011, p. 7-10.

22. Le Roy LadurIE, Emmanuel, Histoire du climat..., op. cit. Tome 1.

23. RouSSEAU, D., "Les températures mensuelles en région parisienne de 1676 à 2008 ", La météorologie, $8^{\mathrm{e}}$ série, $\mathrm{n}^{\circ}$ 67, 2009, p. 43-55.

24. Angot, Alphonse, Le cidre, son introduction dans le pays de Laval, 1889, Archives départementales de la Mayenne, [http://www.lamayenne.fr], 6 p.; MussET, René, "Les limites de la vigne dans l'ouest de la France ", Annales de géographie, 1908, p. 268-270. Id., Le Bas Maine, étude géographique, Paris, Armand Colin, 1917; MARCHAND, Jean-Pierre, Bonnardot, Valérie, Planchon, Olivier, Dubreuil, Vincent, " La vigne, le vin et le climat vu par un notable lavallois au début du XvI $\mathrm{e}^{\mathrm{e}}$ siècle ", Actes du XXIV colloque AIC, Rovereto, Université de Ferrare, 2011, p. 381-386.

25. LE MEné, Michel, Les campagnes angevines..., op. cit.

26. CRoIx, Alain, La Bretagne aux $16^{e}$ et $17^{e}$ siècles. La vie-la mort-la foi, 2 tomes, Paris, Maloine, 1981.

27. GARNIER, Emmanuel, Les dérangements du temps, 500 ans de chaud et de froid en Europe, Paris, Plon, 2010. 
une seule chronique assimilable à un livre de raison, puzzle qu'il va falloir déchiffrer.

Les historiens ont tendance à considérer qu'en absence de renseignements, l'année, ou la saison, relève de la normalité climatique, sans que cette dernière soit jamais définie, et que seuls les aléas susceptibles d'obérer les récoltes sont signalés dans les livres de raison et dans les registres paroissiaux ${ }^{28}$. J.-P. Marchand (1981) a montré, à partir de la série de 139 ans de Dublin Phoenix Park ${ }^{29}$, qu'en climat océanique l'organisation des rythmes pluviométriques mensuels regroupait quatorze types d'organisation différents et que le régime moyen n'était qu'une approximation en climat tempéré océanique ${ }^{30}$.

Or, en l'absence de données climatiques chiffrées ou de descriptions précises, on peut cerner l'évolution du climat à partir de la qualité des récoltes et du prix des céréales. Nombre d'historiens, comme E. Le Roy Ladurie, F. Lebrun, A. Croix, tout en émettant des réserves tenant compte à la fois du contexte politique et des manipulations monétaires ${ }^{31}$, se servent des prix du blé comme marqueur du climat en utilisant des mercuriales qui sont absentes de la chronique de Le Doyen, puisque si celui-ci nous donne presque chaque année les prix du blé (figure 1), il ne s'agit que de notations personnelles, non de véritables mercuriales.

Outre le fait que les unités monétaires et de poids peuvent être variables d'une année à l'autre, Le Doyen nous fournit souvent deux prix. L'un d'avant la moisson traduit la rémanence de l'année précédente avec des problèmes de soudure, d'accaparement ou d'importations de Bretagne ou de Beauce : c'est donc un prix " politique ". L'autre, donné après les battages, est plus lié au climat du printemps et de l'été; le critère choisi ici est alors la qualité de la récolte plus que sa valeur en argent. De plus, cette qualité traduit évidemment le bilan climatique des saisons qui précèdent, tout comme éventuellement l'arrivée brutale pendant la moisson d'un orage qui peut ruiner les effets d'un printemps et d'un été favorables à la croissance du blé. E. Le Roy Ladurie met en garde également sur les cas d'échaudage qui, avec des pics de chaleur au printemps, entraînent des récoltes médiocres ${ }^{32}$.

La courbe des prix du blé telle qu'elle a été reconstituée grâce aux indications de Le Doyen (figure 1) n'a pas servi à définir le climat, mais plutôt à vérifier a posteriori si le climat avait une part dans l'évolution des prix.

28. LEBRun, François, Les hommes et la mort en Anjou aux $17^{e}$ et $18^{e}$ siècles, ParisLa Haye, Mouton, 1971. Le Roy LAdurie, Emmanuel, Histoire humaine..., op. cit.

29. MARCHAND, Jean-Pierre, "La variabilité de l'organisation mensuelle des précipitations. L'exemple de Dublin Phoenix Park 1838-1976 ", Eaux et climats, Grenoble, 1981, p. 325-338.

30. MARchand, Jean-Pierre, Contraintes climatiques et espace géographique, le cas Irlandais, Caen, Paradigme, 1985.

31. BoIs, Guy, "Le prix du froment à Rouen au Xve siècle ", Annales ESC, 1968, vol. 6, p. 1280. Crolx, Alain, La Bretagne aux $16^{e}$ et $17^{e}$ siècles..., op. cit.

32. LE Roy LADURIE, Emmanuel, Histoire humaine..., op. cit. 
Figure 1 - Prix du blé à Laval selon Guillaume Le Doyen (écarts à la moyenne 1481-1537)

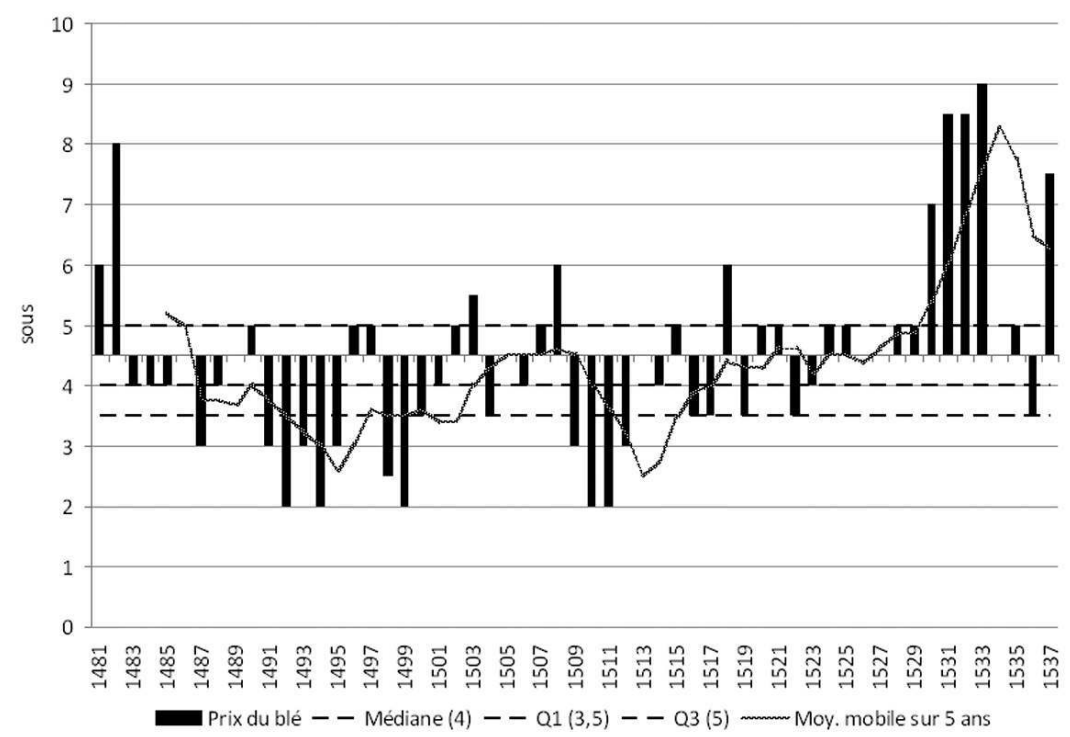

Ainsi, les années 1529-1534 sont caractérisées par des cours du blé élevés quand alternent à Laval des printemps pluvieux (1531) et la sécheresse (1533) (tableau 5, infra). Mais Le Doyen souligne en parallèle la pauvreté et la disette, la peste (1530) ainsi que la guerre et l'accaparement réalisé par les bourgeois de la ville (1530) qui expliquent en partie la hausse du coût de la vie.

La reconstitution de séries climatiques issues de données textuelles suppose que l'on définisse le pas de temps selon lequel vont être étudiées ces séries. A. Croix a eu recours à un pas de temps mensuel ${ }^{33}$ pour sa reconstitution du climat lavallois qui utilise les informations apportées par Le Doyen. Il a défini deux classes pour les précipitations et températures : supérieure ou inférieure, supposant implicitement qu'il y avait là une sorte de moyenne vécue, perçue par Le Doyen. Mais est-ce bien le temps perçu, dominant, qui est pris en compte chez A. Croix et tel qu'a pu le vivre Le Doyen ou, comme le dit l'auteur en évoquant sa propre analyse : " c'est mon climat ", réduit ainsi à une approche des aléas?

L'autre biais chez A. Croix est de proposer le climat de Laval comme représentatif du climat de la Bretagne, surtout en prenant le mois comme unité temporelle, ce qui suppose chez l'auteur une hypothèse forte sur l'homogénéité du climat océanique de la France de l'Ouest d'une part, et

33. Crolx, Alain, La Bretagne aux $16^{e}$ et $17^{e}$ siècles..., op. cit. 
de sa faible variabilité spatio-temporelle d'autre part, ce qui reste assez discutable ${ }^{34}$.

Pour sa part, dans son utilisation de la chronique de Le Doyen, M. Le Mené oppose les hivers froids aux hivers doux en extrapolant le climat probable de Laval à l'Anjou, mais rajoute les dates de vendanges angevines ainsi que la présence des glaces en Loire ${ }^{35}$.

\section{Aléa hivernal et vulnérabilité de printemps et d'été}

Si tous les ans, ou presque, Le Doyen évoque, directement ou indirectement, le climat régnant à Laval, il ne le décrit pas de la même façon pour l'hiver et les autres saisons. L'hiver est saisi par l'aléa : "Au regard de l'yver, il fist si aspre/que le peuple occist de la froidure qu'il faisoit/en nèges tout en surmontoit " ou, à l'opposé, " hyver fût assez courtois et amoureux, mout joyeux " (1507). De telles notations existent pour les autres saisons mais elles sont relativement rares par rapport à celles enregistrées pour l'hiver. Pour le printemps et l'été, ce n'est plus l'aléa qui est dominant dans les écrits de Le Doyen mais la vulnérabilité. En effet, la qualité des blés et du vin, des récoltes et des vendanges sont les critères retenus ici pour cerner la variabilité du climat :
" De blé il en fut à foison
Et cueilli de bonne saison
Il fût aussi bon vin nouveau
Et pour le peuple réjoui
La chair fût à vil prix » (1492)
"Avril fust maulvais garczon
Si fut may car tout y gela,
Vignes arbres, tout ce vola " (1517)

Outre les remarques sur les vendanges, les notations sur le temps et ses conséquences sont absentes de l'automne au sens où on l'entend actuellement, alors que le printemps et l'été sont bien identifiés. Mais, comme le souligne E. Le Roy Ladurie ${ }^{36}$, le début de l'hiver n'a pas la même signification climatologique qu'actuellement et cette saison commence souvent avec l'arrivée du mauvais temps. C'est ainsi que G. Le Doyen décrit en hiver de fortes précipitations et le froid à la Saint-Martin (1523), donc en novembre. L'automne est ainsi assimilé à une "saison froide " et souvent humide, et souvent intégré à l'hiver. Au risque de l'actualisme mais aussi pour pouvoir tester l'hypothèse de la rémission du petit âge glaciaire et faire des comparaisons avec des époques plus tardives, on a pris le parti ici

34. DuRAND-DASTES, François et SANDERS, Lena, " Les contrastes climatiques : Noyaux et zones de transition " dans QuANT, Théo (dir.), Géoscopie de la France, Paris, Minard, 1984, p. 91-101.

35. LE MENÉ, Michel, Les campagnes angevines..., op. cit.

36. Le Roy LadurIE, Emmanuel, Histoire humaine..., op. cit. 
de se caler le plus possible sur les saisons actuelles en joignant l'automne à l'hiver, étant donné la rareté des données automnales.

\section{La mesure de l'incertitude}

Qu'en hiver, on décrive le climat lavallois à partir de séquences de types de temps vécues, et qu'au printemps et en été on l'estime par des bilans socio-économiques, il s'agit donc essentiellement d'interprétations à dire d'expert que l'on doit estimer par un avis autorisé quant à l'exactitude de ces données. Cette question fondamentale de l'évaluation a été abordée par Emmanuel Garnier ${ }^{37}$ qui propose plusieurs indices sur la gravité et l'intensité des phénomènes, aléas et vulnérabilités, selon une démarche initiée par Van Engelen ${ }^{38}$, mais on peut aussi chercher à cerner l'incertitude liée à ces interprétations.

Si l'imprécision relève des données, l'incertitude traduit un état de doute lié à l'observateur et à la façon dont il recueille et traite les données. Or, ici, l'incertitude est double car les observateurs, les experts sont à deux niveaux. Le premier est celui de la mémoire de Le Doyen : quelles étaient ses préoccupations pour une année donnée? Le climat était-il à ses yeux prioritaire par rapport aux autres événements de la ville, comme l'entrée du roi, les pestes ou les mystères? Ce n'est qu'en 1481 et en 1513 que la chronique débute par le climat. Toutefois, il semble que de 1480 à 1500 environ la rédaction ait été réalisée postérieurement, au contraire de la fin de la chronique rédigée plus au fil de la plume d'oie ${ }^{39}$. Le second niveau est celui des auteurs de cet article, de leur lecture et de leur méthodologie consistant à extrapoler les qualités des récoltes du blé et celles des vendanges pour le printemps et l'été et à passer d'une séquence vécue à toute une saison pour l'hiver. Il est donc nécessaire de quantifier cette incertitude et de fournir une échelle de confiance pour chaque estimation.

Pour cela on a adapté sur une échelle de 1 à 5 les propositions du GIEC ${ }^{40}$ pour "l'estimation de l'incertitude des données qualitatives et fondée sur un avis autorisé quant à l'exactitude des données en employant un degré de confiance pour exprimer la probabilité qu'une conclusion est correcte».

À chaque étape de la recherche, pour chaque interprétation saisonnière, ces degrés ont été établis de façon à exprimer la double confiance exprimée vis-à-vis des écrits de Le Doyen et celle de nos propres interprétations. On les retrouve dans le tableau de synthèse en regard de chacune des évaluations saisonnières ( $c f$. infra, Tableau 5).

37. GARNIER, Emmanuel, Les dérangements du temps..., op. cit.

38. VAn Engelen, A. F. V., Le Climat du dernier millénaire en Europe (accès en ligne : [www. knmi.nl/publications/fulltexts/climatduderniermillnaire.pdf]), 2001.

39. LE MENÉ, Michel, Les campagnes angevines..., op. cit.

40. GIEC, Bilan 2007 des changements climatiques. Contribution des Groupes de travail I, II et III au quatrième Rapport d'évaluation du Groupe d'experts intergouvernemental sur l'évolution du climat, publié sous la direction de, PAchauri, R.K. et REISINGER, A., Genève, GIEC, 2007. 


\section{Tableau 1 - Degrés et indices de confiance pour des données quali- tatives}

\begin{tabular}{|l|l|}
\hline Degré de confiance & indice \\
\hline très élevé & 5 \\
\hline élevé & 4 \\
\hline moyen & 3 \\
\hline faible & 2 \\
\hline très faible & 1 \\
\hline
\end{tabular}

\section{Les types de temps vécus probables à Laval (1481-1537)}

L'étude du climat de Laval pendant cette période repose sur l'analyse des types de temps, c'est-à-dire des " ambiances climatiques " vécues par une population et que l'on peut décrire par les agents du climat que sont le vent, la pluie, la température etc.

\section{Les types de temps hivernaux}

L'analyse des situations hivernales décrites dans le tableau $n^{\circ} 2{ }^{41}$ pour la période 1481-1537 montre que Le Doyen nous donne des chroniques climatiques saisonnières par séquences de types de temps. Les types de temps dominants correspondent à des séquences froides ou douces, suffisamment longues pour que Le Doyen les ait vécues comme telles.

La succession des hivers ne se marque pas à Laval par des périodes froides continues, bien que les années 1510-1524 aient connu une séquence de ce type. Les périodes douces liées à des types de temps océanique bien connus dominent ${ }^{42}$.

Même si ce tableau n'indique pas les très grands froids, ceux qui font geler les vignes $(1481,1511)$ et qui sont statistiquement en bout de distribution, on peut noter que les situations de grand froid ou de beau temps sont les plus fréquentes au détriment des "situations intermédiaires". Les types (T1, T2, T8, T9) ont, par leur intensité et leurs séquences, fortement marqué les esprits lavallois, d'autant que la fréquence de certains hivers qui perdurent au-delà de Pâques indique une probabilité décennale de subir un hiver long et dur.

On touche ici une autre limite de la recherche. Le climat vécu tel qu'il est décrit par Le Doyen est celui qui a le plus frappé l'auteur et ses concitoyens, pour l'année en question et non par analyse fréquentielle des situations même perçues. Mais cette perception du climat vécu a-t-elle profondément changé depuis 500 ans?

41. MARChAnd, Jean-Pierre, Planchon, Olivier, BonnARdot Valérie, " Le climat vécu, une aide à la détermination des climats passés : les hivers à Laval (1481-1537) ", Actes du XXIII colloque AIC, Rennes, 2010, p. 373-378.

42. Mounier, Jean, Les climats océaniques des régions atlantiques de l'Espagne et du Portugal, Lille, Atelier de reproduction des thèses, 2 tomes + atlas, 1979. 


\section{Tableau 2 - Description des types de temps hivernaux vécus et circulation atmosphérique probable (1481-1537)}

\begin{tabular}{|c|c|c|c|c|}
\hline & $\begin{array}{c}\text { Séquences de type de temps } \\
\text { vécus }\end{array}$ & $\begin{array}{c}\text { Type de circulation atmosphé- } \\
\text { rique probable }\end{array}$ & Occurrences & $\%$ \\
\hline T1 & $\begin{array}{c}\text { Âpres et froids, longs sans } \\
\text { grêle, neige ou pluie }\end{array}$ & $\begin{array}{c}\text { Anticyclonique d'est et nord- } \\
\text { est }\end{array}$ & 11 & 25 \\
\hline T2 & $\begin{array}{c}\text { Id. mais durent au-delà de } \\
\text { Pâques }\end{array}$ & $\begin{array}{c}\text { Anticyclonique d'est et nord- } \\
\text { est }\end{array}$ & 4 & 9 \\
\hline T3 & Froid avec glace, pluie, neige & Circulation de nord & 2 & 5 \\
\hline T4 & Neige couvrant le sol & Anticyclonique avec air froid & 3 & 4 \\
\hline T5 & Froids et vents, grands vents & Perturbé d'ouest, nord-ouest & 1 & 2 \\
\hline T6 & Doux et pluvieux & Perturbé de sud-ouest & 5 & 12 \\
\hline T7 & $\begin{array}{c}\text { Ni trop fort ni frais, tantôt } \\
\text { humide, tantôt sec }\end{array}$ & $\begin{array}{c}\text { Forte variabilité. Dominante de } \\
\text { flux d'ouest }\end{array}$ & 2 & 2 \\
\hline T8 & $\begin{array}{c}\text { Assez convenable; pas de } \\
\text { gelées }\end{array}$ & $\begin{array}{c}\text { Variabilité moyenne. Flux } \\
\text { d'ouest/sud-ouest dominant }\end{array}$ & 11 & 25 \\
\hline T9 & $\begin{array}{c}\text { "Assez courtois et amoureux } \\
\text { moult joyeux ". Pas de gelées } \\
\text { ni de crues ni de neige }\end{array}$ & $\begin{array}{c}\text { Anticyclonique de sud-ouest } \\
\text { et Flux faiblement perturbé } \\
\text { d'ouest }\end{array}$ & 6 & 14 \\
\hline
\end{tabular}

\section{Les types de temps de printemps et d'été}

Pour le printemps et l'été, la méthodologie n'est pas du même ordre, les données chiffrées étant toujours absentes. Le Doyen, contrairement à ce qu'il fait pour l'hiver, fournit peu de renseignements sur le climat vécu au printemps et en été, s'attachant plutôt à décrire des phénomènes exceptionnels datés, comme de fortes gelées ou des tempêtes susceptibles d'avoir une influence sur les récoltes.

Certes, certaines années particulièrement humides ou très sèches (1533) sont signalées mais le corpus est insuffisant pour qu'on puisse en tirer des conclusions sur les séquences significatives, comme cela a été le cas pour l'hiver. Par contre, les données en quantité et qualité sur les récoltes de blé et de vin sont suffisamment nombreuses pour que l'on puisse extrapoler le temps probable des saisons concernées. Pour ce qui concerne les vendanges, Le Doyen note non seulement la qualité et la quantité du vin récolté aux alentours de Laval, mais il indique aussi les importations de vin en provenance de l'Anjou ou d'Orléans, vins d'une qualité supérieure, selon ses dires, à celle du vin lavallois : " Peu en avait été cueilli/ En ce pays et mal recueilly/Mais d'Orléans nous eusmes vins/Qui étaient très bons et très fins " (1496).

Une première phase a consisté à établir le fichier de base, sans chercher à interpréter ni simplifier le vocabulaire de Le Doyen, et à fournir une première évaluation de l'incertitude à dire d'expert. Or ce vocabulaire est riche, le choix pour Le Doyen de s'exprimer en vers n'en facilitant pas l'harmonisation! Pour qualifier les qualités et les quantités des blés, le vocabu- 
laire est moins varié que pour décrire la qualité du vin, sujet éminemment sensible chez lui.

\title{
Tableau 3 - Vocabulaire utilisé par Le Doyen pour décrire la qualité du vin et des vendanges
}

\begin{abstract}
Peu de vin bonne qualité - Année fructueuse - Vins trop verts - Très bonne qualité - Bon vin nouveau - Bon vin nouveau et pas très fort - En quantité mais peu cher - Import de vin - Vin médiocre - peu de grains cueillis (très bon vin d'Orléans) Vignes grêlées en juin - Vin peu cher - En quantité et bon marché (pas d'import) - Suffisamment de vin - Bon vin de Saint-Melaine (proche Saint-Denis) - Bon vin mais peu cueilli - Bon vin en saison - Vin poussèrent mais de mauvaises qualité. Vins verts qui servent de verjus - Pauvre louange (local et d'Orléans) - Peu de vin cueilli. Bon vin de Saint-Denis - Très bonnes vendanges - Pauvres louanges - Louanges pour vendanges pas grande abondance. Cidre remplace vin - Vin commun en qualité suffisante - Vin en quantité et en saison - Vin moyen, faible récolte - Vins chers (gelées de printemps) - Bon vin de Saint-Denis - Vin de toutes les couleurs qui fait mal aux dents. Import d'Orléans - Vin et cidre grande abondance - Louange à Dieu - Peu de vin mais bien recueilli - Peu de vin de pays et médiocre - Vin et cidre à foison - Assez de vin de bonne qualité - Pauvre vin local, récolte médiocre - Import d'Orléans et Montrichard - " Non fut en vin et autre boire que vin je grippe " - Import de Fromentières - Peu de vin mais bon pour l'été. Importation du Layon.
\end{abstract}

Dans une deuxième phase, le vocabulaire a été harmonisé pour déboucher sur des critères climatiques homogénéisés. Toutefois la vigne a demandé une étape intermédiaire qui fournit déjà des indications sur le climat du printemps et surtout de l'été.

\section{Tableau 4 - Vocabulaire harmonisé relatif à la qualité du vin et occurrences (1480-1537)}

\begin{tabular}{|c|c|c|}
\hline $\begin{array}{c}\text { Termes descriptifs relatifs } \\
\text { au vin }\end{array}$ & Années & Total \\
\hline Pas de vin (gelées) & $1481-1490-1517-1522-1524-1532$. & 6 \\
\hline Vin médiocre & $1482-1496-1506-1511-1523-1526-1529-1535-$ & 10 \\
\hline Vin trop vert & $1536-1537$ & 3 \\
\hline Peu mais honnête & $1488-1505-1519$ & 1 \\
\hline Vin commun & 1507 & 4 \\
\hline Bonne quantité & $1483-1484-1487-1494-1498-1499-1509-1514-$ & 10 \\
\hline Bonne qualité & $1491-1492-1504-1509-1528-1530-1531-1532-1534$ & 9 \\
\hline Bon mais peu & $1485-1486-1501-1502-1512-1521-1527$ & 7 \\
\hline Bon mais pas trop fort & 1493 & 1 \\
\hline Peu cher & 1497 & 1 \\
\hline
\end{tabular}


L'évolution saisonnière du climat à Laval (1481-1537)

Le tableau 5 donne l'interprétation climatique finale, assortie des indices de confiance pour chaque saison telle qu'elle apparaît chez Le Doyen.

\section{Tableau 5 - Chronique des types de temps vécus à Laval (1481-1537) par saison (Années manquantes en grisé)}

\begin{tabular}{|c|c|c|c|c|c|c|}
\hline \multirow{2}{*}{ années } & \multicolumn{5}{|c|}{ Description du temps et indice de confiance (IC) par saison } & \multirow[b]{2}{*}{ IC } \\
\hline & HIVER & IC & PRINTEMPS & IC & ÉTÉ & \\
\hline 1481 & $\begin{array}{l}\text { Très froid et } \\
\text { long } \\
\text { Crue de } \\
\text { débâcle. }\end{array}$ & 5 & Plutôt ensoleillé & 2 & $\begin{array}{l}\text { Pas de raisin } \\
\text { (Blés exportés } \\
\text { en Bretagne) }\end{array}$ & 0 \\
\hline 1482 & Plutôt doux & 3 & Humide et frais & 3 & Frais et humide & 3 \\
\hline 1483 & $\begin{array}{l}\text { Doux et peu } \\
\text { humide }\end{array}$ & 4 & Humide et frais & 3 & Ensoleillé & 4 \\
\hline 1484 & $\begin{array}{l}\text { Une alternance } \\
\text { de temps } \\
\text { perturbé et de } \\
\text { grands froids }\end{array}$ & 3 & Plutôt ensoleillé & 3 & $\begin{array}{l}\text { Août ensoleillé } \\
\text { Été ensoleillé }\end{array}$ & $\begin{array}{l}3 \\
3\end{array}$ \\
\hline 1485 & & & Chaud et humide & 3 & $\begin{array}{l}\text { Août sec et } \\
\text { ensoleillé }\end{array}$ & $2 / 3$ \\
\hline 1486 & & & $\begin{array}{c}\text { Humide } \\
\text { (sauf échaudage) }\end{array}$ & 3 & Sec et chaud & 4 \\
\hline 1487 & & & $\begin{array}{l}\text { Ensoleillé et peu } \\
\text { humide }\end{array}$ & 3 & $\begin{array}{c}\text { Chaud avec } \\
\text { passages } \\
\text { humides (?) }\end{array}$ & $\begin{array}{l}3 \\
2\end{array}$ \\
\hline 1488 & & & $\begin{array}{l}\text { Ensoleillé, peu } \\
\text { humide }\end{array}$ & 3 & Froid et humide & 4 \\
\hline 1489 & Très froid et sec & 4 & Humide et frais & 4 & Froid et humide & 4 \\
\hline 1490 & $\begin{array}{c}\text { Pas de } \\
\text { séquences } \\
\text { froides ou très } \\
\text { pluvieuses }\end{array}$ & 3 & $\begin{array}{l}\text { Des gelées de } \\
\text { printemps, mais } \\
\text { avec soleil }\end{array}$ & 4 & $?$ & \\
\hline 1491 & Sec et doux & 2 & $\begin{array}{c}\text { Ensoleillé et } \\
\text { chaud } \\
\text { (sans excès) }\end{array}$ & 4 & Sec et chaud & 4 \\
\hline 1492 & & & $\begin{array}{c}\text { Ensoleillé et peu } \\
\text { humide }\end{array}$ & 4 & Sec et chaud & 4 \\
\hline 1493 & & & $\begin{array}{c}\text { Ensoleillé et } \\
\text { chaud sans excès } \\
\text { (pas d'échau- } \\
\text { dage) }\end{array}$ & 4 & Chaud & 4 \\
\hline 1494 & $\begin{array}{c}\text { Très humide } \\
\text { Froid (? pesant) }\end{array}$ & $\begin{array}{l}4 \\
2\end{array}$ & $\begin{array}{l}\text { Sec et chaud sans } \\
\text { excès }\end{array}$ & 4 & $\begin{array}{l}\text { Août assez } \\
\text { chaud } \\
\text { et humide à } \\
\text { la fin }\end{array}$ & $\begin{array}{l}3 \\
3\end{array}$ \\
\hline
\end{tabular}




\begin{tabular}{|c|c|c|c|c|c|c|}
\hline 1495 & $\begin{array}{l}\text { Plutôt doux et } \\
\text { peu humide }\end{array}$ & 3 & $\begin{array}{c}\text { Chaud et sec sans } \\
\text { excès }\end{array}$ & 3 & $\begin{array}{l}\text { Juillet sec } \\
\text { Août humide et } \\
\text { frais }\end{array}$ & \\
\hline 1496 & Très humide & 5 & $\begin{array}{l}\text { Chaud puis } \\
\text { orages } \\
\text { fin juin }\end{array}$ & 4 & Sec et chaud & 2 \\
\hline 1497 & Froid et sec & 4 & $\begin{array}{l}\text { Plutôt humide } \\
\text { puis chaud } \\
\text { Ou chaud et } \\
\text { humide }\end{array}$ & 2 & Assez chaud & 3 \\
\hline 1498 & Froid et humide & 4 & Chaud sans excès & 4 & Chaud et sec & 4 \\
\hline 1499 & $\begin{array}{c}\text { Doux } \\
\text { et peu humide }\end{array}$ & $\begin{array}{l}4 \\
2\end{array}$ & Chaud sans excès & 4 & Chaud et sec & 3 \\
\hline 1500 & Très froid et sec & 4 & $\begin{array}{l}\text { Assez frais et } \\
\text { humide (?) cf. } \\
\text { soudure } 1501\end{array}$ & 2 & Chaud et sec & 4 \\
\hline 1501 & $\begin{array}{l}\text { Doux } \\
\text { et peu humide }\end{array}$ & $\begin{array}{l}4 \\
3\end{array}$ & $\begin{array}{l}\text { Chaud mais avec } \\
\text { grêle } \\
\text { en juin }\end{array}$ & 3 & Chaud et sec & 4 \\
\hline 1502 & $\begin{array}{l}\text { Hiver doux et } \\
\text { fort pluvieux }\end{array}$ & $4 / 5$ & $\begin{array}{l}\text { Frais et peu plu- } \\
\quad \text { vieux (?) }\end{array}$ & $\begin{array}{l}3 \\
2\end{array}$ & Chaud et sec & 3 \\
\hline 1503 & $\begin{array}{l}\text { Doux et } \\
\text { agréable }\end{array}$ & 2 & $\begin{array}{c}\text { Frais et humide } \\
\text { (?) }\end{array}$ & 2 & $?$ & $\mathrm{XX}$ \\
\hline 1504 & $\begin{array}{l}\text { Doux et peu } \\
\text { humide }\end{array}$ & 3 & Chaud sans excès & 3 & $\begin{array}{c}\text { Chaud et très } \\
\text { sec }\end{array}$ & 4 \\
\hline 1505 & $\begin{array}{l}\text { Doux et peu } \\
\text { humide }\end{array}$ & 4 & $\begin{array}{c}\text { Printemps froid et } \\
\text { humide } \\
\text { (mai et juin plu- } \\
\text { vieux) }\end{array}$ & $\begin{array}{l}3 \\
4\end{array}$ & $\begin{array}{l}\text { Juillet pourri } \\
\text { Bels août et } \\
\text { septembre }\end{array}$ & $\begin{array}{l}5 \\
4\end{array}$ \\
\hline 1506 & $\begin{array}{l}\text { Froid dès la fin } \\
\text { de l'automne et } \\
\text { neige; crue de } \\
\text { débâcle et de } \\
\text { fonte puis pluie }\end{array}$ & $\begin{array}{l}5 \\
3\end{array}$ & $\begin{array}{l}\text { Avril, mai cor- } \\
\text { rects } \\
\text { Juin très humide }\end{array}$ & 5 & $\begin{array}{l}\text { Possibilité } \\
\text { d'orages } \\
\text { Pluvieux en } \\
\text { juillet } \\
\text { et août }\end{array}$ & 4 \\
\hline 1507 & $\begin{array}{l}\text { Doux et peu } \\
\text { humide }\end{array}$ & 5 & Frais et humide & 4 & Frais et humide & 4 \\
\hline 1508 & $\begin{array}{c}\text { Doux et assez } \\
\text { sec }\end{array}$ & 4 & Chaud sans excès & 4 & Chaud et sec & 2 \\
\hline 1509 & $\begin{array}{l}\text { Long, sec et } \\
\text { froid }\end{array}$ & 5 & $\begin{array}{c}\text { Humide puis } \\
\text { chaud et sec sans } \\
\text { excès }\end{array}$ & 4 & $\begin{array}{l}\text { Chaud et sec, } \\
\text { jusqu'à l'au- } \\
\text { tomne }\end{array}$ & 5 \\
\hline 1510 & Long et froid & 5 & $\begin{array}{l}\text { Mai, juin, juillet : } \\
\text { chaud et sec }\end{array}$ & 4 & Beau et sec & 4 \\
\hline 1511 & $\begin{array}{l}\text { Long âpre et } \\
\text { froid (Gelées à } \\
\left.\qquad-15^{\circ}\right)\end{array}$ & 5 & $\begin{array}{l}\text { Froid au début } \\
\text { puis plus chaud } \\
\text { et sec jusqu'à la } \\
\text { moisson. Mais } \\
\text { tout est retardé }\end{array}$ & 3 & $\begin{array}{l}\text { Août ordinaire } \\
\text { sans excès } \\
\text { Octobre : cor- } \\
\text { rect Novembre : } \\
\text { très humide }\end{array}$ & $\begin{array}{l}2 \\
4\end{array}$ \\
\hline
\end{tabular}




\begin{tabular}{|c|c|c|c|c|c|c|}
\hline 1512 & $\begin{array}{l}\text { Long âpre et } \\
\text { froid }\end{array}$ & 5 & $\begin{array}{l}\text { Début froid et } \\
\text { humide puis }\end{array}$ & 4 & Chaud et sec & 4 \\
\hline 1513 & $\begin{array}{l}\text { Pluie et vent } \\
\text { jusqu'à mi-jan- } \\
\text { vier puis froid } \\
\text { et sec }\end{array}$ & 4 & $\begin{array}{l}\text { Froid jusque } \\
\text { vers début mai } \\
\text { Puis doux et } \\
\text { humide }\end{array}$ & $\begin{array}{l}3 \\
2\end{array}$ & $\begin{array}{l}\text { Chaud et assez } \\
\text { humide. }\end{array}$ & 3 \\
\hline 1514 & & 5 & $\begin{array}{l}\text { Froid en avril puis } \\
\text { chaud sans excès }\end{array}$ & 4 & $\begin{array}{l}\text { Chaud et un } \\
\text { peu humide (en } \\
\text { août?) }\end{array}$ & 3 \\
\hline 1515 & $\begin{array}{l}\text { Doux et peu } \\
\text { pluvieux }\end{array}$ & 5 & Frais et humide & 4 & Frais et humide & 4 \\
\hline 1516 & $\begin{array}{l}\text { Ni froid, ni } \\
\text { mauvais, } \\
\text { ni gelée, ni } \\
\text { neige }\end{array}$ & 5 & $\mathrm{Sec}$ & 4 & & \\
\hline 1517 & $\begin{array}{l}\text { Plutôt sec et } \\
\text { froid }\end{array}$ & 3 & $\begin{array}{l}\text { Avril, mai très } \\
\text { froids. Puis froid } \\
\text { et pluvieux }\end{array}$ & 4 & $\begin{array}{l}\text { Froid et plu- } \\
\text { vieux, } \\
\text { jusqu'en } \\
\text { automne. }\end{array}$ & 4 \\
\hline 1518 & $\begin{array}{c}\text { Hiver long, } \\
\text { humide et ven- } \\
\text { teux en mars } \\
\text { avril } \\
\end{array}$ & $\begin{array}{l}2 \\
4\end{array}$ & $\begin{array}{l}\text { Avril avec temps } \\
\text { perturbé, puis } \\
\text { chaud sans excès }\end{array}$ & 4 & $\begin{array}{l}\text { Assez chaud et } \\
\text { sec }\end{array}$ & 2 \\
\hline 1519 & $\begin{array}{c}\text { Hiver assez } \\
\text { doux, au moins } \\
\text { à la fin }\end{array}$ & $\begin{array}{l}3 \\
4\end{array}$ & $\begin{array}{l}\text { Avril pluvieux } \\
\text { Mai, juin chauds }\end{array}$ & $\begin{array}{l}5 \\
4\end{array}$ & $\begin{array}{l}\text { Tempêtes et } \\
\text { pluies en août } \\
\text { Été frais }\end{array}$ & $\begin{array}{l}4 \\
3\end{array}$ \\
\hline 1520 & \begin{tabular}{|c|} 
Sec en janvier \\
Humide en mars
\end{tabular} & $\begin{array}{l}3 \\
4\end{array}$ & Humide et frais & 3 & $\begin{array}{l}\text { Chaud et un peu } \\
\text { humide (août) }\end{array}$ & 4 \\
\hline 1521 & $\begin{array}{l}\text { Ni trop froid, ni } \\
\text { trop humide }\end{array}$ & $2 / 3$ & $\begin{array}{l}\text { Frais et humide } \\
\text { (Blé?) }\end{array}$ & 2 & $\begin{array}{l}\text { Chaud et sec } \\
\text { Septembre beau }\end{array}$ & 4 \\
\hline 1522 & $\begin{array}{c}\text { Fin d'automne } \\
\text { doux } \\
\text { Puis très froid } \\
\text { en janvier avec } \\
\text { neige }\end{array}$ & $\begin{array}{l}3 \\
4\end{array}$ & $\begin{array}{l}\text { Peu humide et } \\
\text { chaud }\end{array}$ & 4 & Plutôt beau & 2 \\
\hline 1523 & & & Frais et humide & 3 & $\begin{array}{l}\text { Humide et } \\
\text { froid depuis la } \\
\text { moisson. }\end{array}$ & 5 \\
\hline 1524 & $\begin{array}{c}\text { Précoce. Plutôt } \\
\text { froid }\end{array}$ & 2 & $\begin{array}{c}\text { Chaud et sec avec } \\
\text { orage } \\
\text { (échaudage?) }\end{array}$ & $\begin{array}{l}4 \\
2\end{array}$ & Humide et frais & 4 \\
\hline 1525 & $\begin{array}{l}\text { Ni trop chaud, } \\
\text { ni trop froid. }\end{array}$ & 5 & Chaud sans excès & 3 & Chaud et sec & 5 \\
\hline 1526 & $\begin{array}{l}\text { Ni trop chaud, } \\
\text { ni trop froid }\end{array}$ & 5 & $\begin{array}{l}\text { Ensoleillé et peu } \\
\text { humide }\end{array}$ & 4 & $\begin{array}{c}\text { Chaud } \\
\text { Chaud et } \\
\text { humide en sep- } \\
\text { tembre (?) }\end{array}$ & 2 \\
\hline 1527 & $\begin{array}{l}\text { Doux } \\
\text { et pluvieux }\end{array}$ & $\begin{array}{l}4 \\
2\end{array}$ & Chaud sans excès & 4 & $\begin{array}{l}\text { Chaud et sec } \\
\text { sauf juillet }\end{array}$ & 3 \\
\hline
\end{tabular}




\begin{tabular}{|c|c|c|c|c|c|c|}
\hline 1528 & $\begin{array}{l}\text { Pas trop froid } \\
\text { ni humide }\end{array}$ & $\begin{array}{l}4 \\
2\end{array}$ & \begin{tabular}{|c|} 
Pluies de prin- \\
temps; plutôt \\
frais \\
Des gelées (fruits)
\end{tabular} & 4 & Chaud et sec & 4 \\
\hline 1529 & $\begin{array}{c}\text { Une période } \\
\text { froide (quand?) } \\
\text { Du temps doux } \\
\text { dominant, } \\
\text { grands vents }\end{array}$ & $\begin{array}{l}2 \\
3\end{array}$ & $\begin{array}{c}\text { Chaud sans excès } \\
\text { jusqu'à la mois- } \\
\text { son }\end{array}$ & 4 & $\begin{array}{c}\text { Eté « moult } \\
\text { joyeux " mais } \\
\text { mauvaises ven- } \\
\text { danges } \\
\text { Maladie sur la } \\
\text { vigne? }\end{array}$ & 1 \\
\hline 1530 & $\begin{array}{l}\text { Doux et très } \\
\text { pluvieux (crue } \\
\text { trentennale) }\end{array}$ & 5 & $\begin{array}{c}\text { Doux et peu } \\
\text { humide } \\
\text { sauf peut être en } \\
\text { juin }\end{array}$ & $\begin{array}{l}4 \\
2\end{array}$ & Chaud et sec & 3 \\
\hline 1531 & $\begin{array}{c}\text { "Moult long et } \\
\text { pluvieux" }\end{array}$ & 4 & $\begin{array}{l}\text { Très humide, } \\
\text { pluie et frais }\end{array}$ & 4 & & \\
\hline 1532 & $\begin{array}{l}\text { Doux et peu } \\
\text { humide }\end{array}$ & 4 & $\begin{array}{c}\text { Chaud sans excès } \\
\text { sauf une gelée de } \\
\text { juin } \\
\text { et une tempête }\end{array}$ & 4 & Chaud et sec & 4 \\
\hline 1533 & $\begin{array}{l}\text { Variabilité atlan- } \\
\text { tique }\end{array}$ & 4 & Chaud et très sec & 5 & $\begin{array}{l}\text { Chaud et très } \\
\text { sec }\end{array}$ & 5 \\
\hline 1534 & Long et froid & 4 & $\begin{array}{c}\text { Chaud et sec (Inf. } \\
1533)\end{array}$ & 5 & $\begin{array}{l}\text { Chaud et sec } \\
\text { (inf. 1533) }\end{array}$ & 5 \\
\hline 1535 & & & Très humide & 5 & Humide et frais & 3 \\
\hline 1536 & & & $\begin{array}{l}\text { Doux et peu } \\
\text { humide }\end{array}$ & 3 & $\begin{array}{c}\text { Pas de pluie } \\
\text { mais frais }\end{array}$ & 3 \\
\hline 1537 & & & Doux et plutôt sec & 3 & $\begin{array}{c}\text { Doux, pas de } \\
\text { grosses cha- } \\
\text { leurs. } \\
\text { Fin août et } \\
\text { septembre : } \\
\text { humides et frais }\end{array}$ & 3 \\
\hline
\end{tabular}

C'est à partir de ce tableau que l'on va pouvoir aborder l'étude de la variabilité interannuelle du climat saisonnier en termes de fréquence mais aussi en déterminant des alternances de séquences pluriannuelles de types de temps.

\section{La variabilité interannuelle des types de temps saisonniers}

Pour visualiser cet aspect du climat, le critère choisi repose sur le croisement classique des variables d'humidité et de température, soit 4 items : chaud et sec, chaud et humide, froid et sec, froid et humide, en sachant bien que cette classification doit être adaptée à la spécificité de chaque saison et qu'une sécheresse estivale n'a rien à voir avec un hiver froid et sec. 
Pour le printemps et l'été, on peut représenter graphiquement les alternances saisonnières sur toute la période d'étude en utilisant ces quatre critères (figure 2).

Tableau 6 - Les types de temps (fréquences absolues par saison)

\begin{tabular}{|l|l|l|l|}
\hline & Hiver & Printemps & Été \\
\hline Doux ou chaud (1) et sec & 14 & 33 & 35 \\
\hline Doux et humide & 5 & 7 & 4 \\
\hline Froid et humide & 10 & 17 & 17 \\
\hline Froid ou frais (1) et sec & 13 & 4 & 1 \\
\hline Variabilité océanique & 5 & & \\
\hline
\end{tabular}

(1) Selon la saison.

Figure 2 - Succession probable des années climatiques

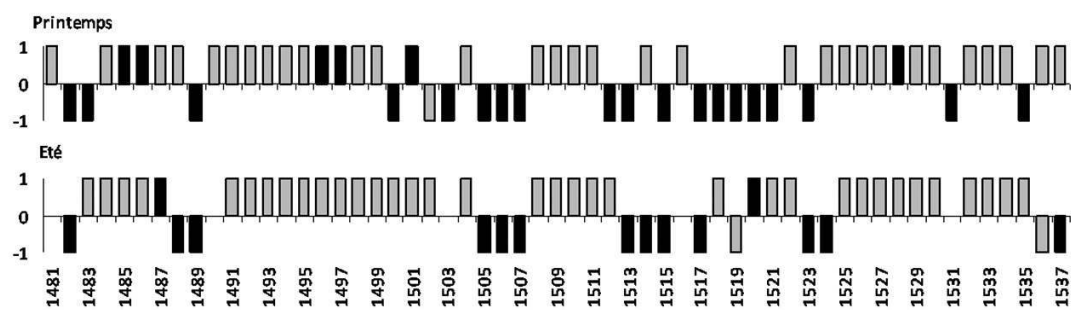

Printemps en haut et été en bas; en positif, les années chaudes, en négatif, les années fraîches; en noir, les années humides et en gris, les années sèches.

\section{La variabilité hivernale}

Dans l'interprétation synoptique du tableau 2, on retrouve les types de circulation atmosphérique du climat tempéré océanique ainsi que les séquences de types de temps dominant dans le vécu et la mémoire des gens de l'ouest de la France et résumant la perception globale d'une saison hivernale. Cette remarque est valable pour le $\mathrm{XvI}^{\mathrm{e}}$ siècle comme de nos jours.

Sous réserve de la surreprésentation des hivers "extrêmes ", qu'ils soient chauds ou froids, le tableau 1 donne une synthèse de la variabilité des types de temps hivernaux. Le tableau 7 montre l'évolution de ces types de temps sur la période allant de 1480 à 1537.

Globalement, les hivers doux semblent un peu plus nombreux que les hivers froids et longs. Les hivers avec séquences vécues de grands froids prolongés représentent 15 cas, soit $33 \%$ de ceux dont le climat est connu.

Si on note une forte variabilité interannuelle, certaines périodes (15101520) apparaissent plus froides que d'autres. Les hivers agréables sont au 
Jean-Pierre MARChAND, Valérie BonnaRdot, Olivier Planchon

Tableau 7 - Types de temps hivernaux vécus de 1481 à 1537

\begin{tabular}{|c|c|c|c|c|c|c|c|c|c|}
\hline \multirow{2}{*}{ Années } & \multicolumn{9}{|c|}{ Types de temps } \\
\hline & $\mathrm{T} 1$ & $\mathrm{~T} 2$ & T3 & $\mathrm{T} 4$ & $\mathrm{~T} 5$ & T6 & $\mathrm{T} 7$ & $\mathrm{~T} 8$ & $\mathrm{~T} 9$ \\
\hline \multicolumn{10}{|l|}{1481} \\
\hline \multicolumn{10}{|l|}{1482} \\
\hline \multicolumn{10}{|l|}{1483} \\
\hline \multicolumn{10}{|l|}{1484} \\
\hline \multicolumn{10}{|l|}{1485} \\
\hline \multicolumn{10}{|l|}{1486} \\
\hline \multicolumn{10}{|l|}{1487} \\
\hline \multicolumn{10}{|l|}{1488} \\
\hline \multicolumn{10}{|l|}{1489} \\
\hline \multicolumn{10}{|l|}{1490} \\
\hline \multicolumn{10}{|l|}{1491} \\
\hline \multicolumn{10}{|l|}{1492} \\
\hline \multicolumn{10}{|l|}{1493} \\
\hline \multicolumn{10}{|l|}{1494} \\
\hline \multicolumn{10}{|l|}{1495} \\
\hline \multicolumn{10}{|l|}{1496} \\
\hline \multicolumn{10}{|c|}{1497} \\
\hline \multicolumn{10}{|l|}{1498} \\
\hline \multicolumn{10}{|l|}{1499} \\
\hline \multicolumn{10}{|l|}{1500} \\
\hline \multicolumn{10}{|l|}{1501} \\
\hline \multicolumn{10}{|l|}{1502} \\
\hline \multicolumn{10}{|l|}{1503} \\
\hline 1504 & & & & & & & & & \\
\hline 1505 & & & & & & & & & \\
\hline 1506 & & & & & & & & & \\
\hline 1507 & & & & & & & & & \\
\hline 1508 & & & & & & & & & \\
\hline 1509 & & & & & & & & & \\
\hline 1510 & & & & & & & & & \\
\hline 1511 & & & & & & & & & \\
\hline 1512 & & & & & & & & & \\
\hline 1513 & & & & & & & & & \\
\hline 1514 & & & & & & & & & \\
\hline 1515 & & & & & & & & & \\
\hline 1516 & & & & & & & & & \\
\hline 1517 & & & & & & & & & \\
\hline
\end{tabular}




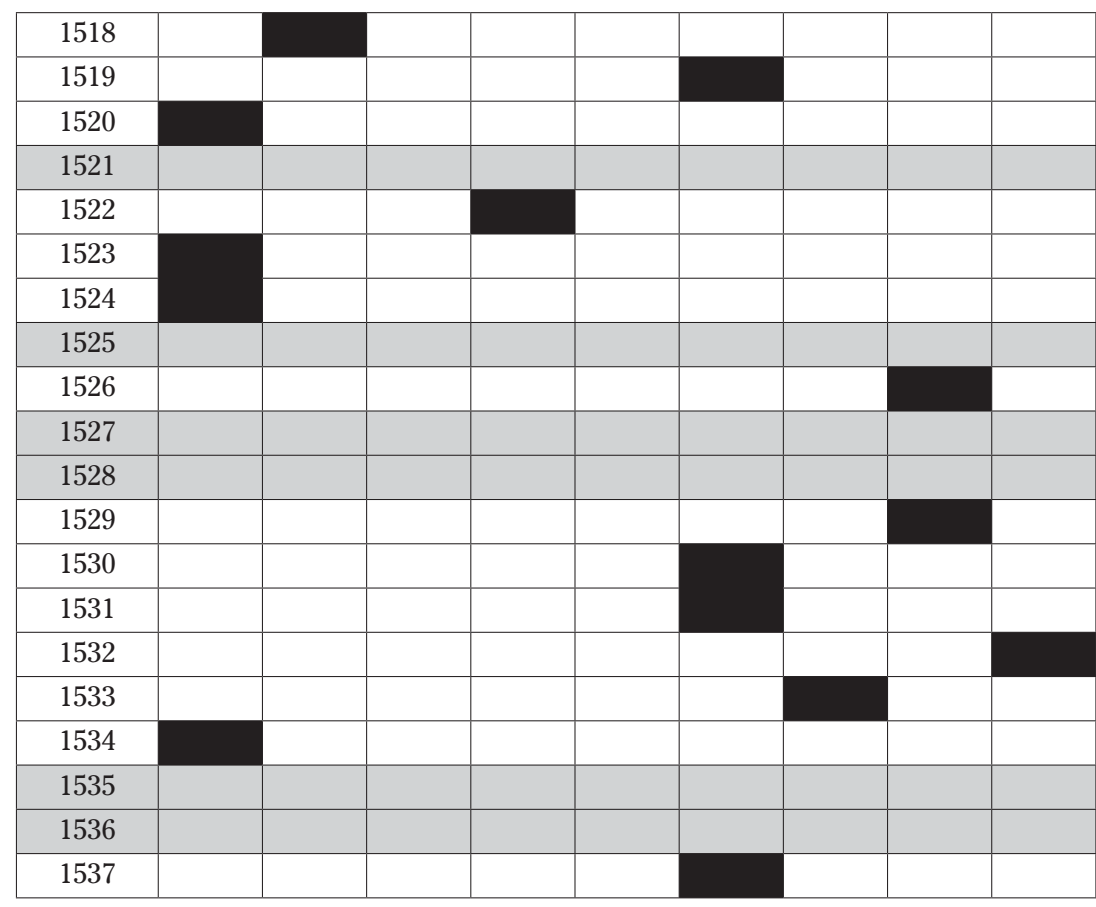

nombre de 16 , soit $39 \%$ des hivers au climat connu. Les années qui vont de 1510 à 1524 sont caractérisées par 10 années particulièrement froides, avec quatre hivers se prolongeant au delà de Pâques. Par contre, les hivers doux (Types 8 et 9) sont plus fréquents dans la période allant de 1490 à 1508 et reviennent à partir de 1526 jusqu'à 1537 . Si l'hiver 1481 semble suivi d'une accalmie hivernale, l'absence de données pour les années 1485 à 1488 ne permet pas de définir une tendance climatique pour la fin de cette décennie. Il en est de même pour 1535 et 1537.

\section{La variabilité au printemps et en été}

La variabilité interannuelle des types de temps indique une dominante des printemps doux et plutôt secs (58 \% des cas) contre 47 \% d'années aux printemps humides affirmés. Pour l'été, la chaleur et la sécheresse sont présentes dans $60 \%$ des cas, mais les étés froids et humides sont présents près d'une année sur trois, ce qui est de mauvais augure pour la qualité et la faisabilité des moissons. À cette variabilité saisonnière, on doit rajouter les jours de grêle (à la Fête-Dieu de 1501) souvent accompagnés de vents violents (18 juin 1496) et d'orages (à la Saint-Jean 1524), voire de gelées tardives comme celle de la Pentecôte 1502, qui rendent délicates l'interprétation saisonnière. La prise en compte de la variabilité interannuelle amène à proposer des séquences pluriannuelles de types de temps dominants. 
Tableau 8 - Séquences pluriannuelles des types de temps de printemps (a) et d'été (b)

\begin{tabular}{|c|c|c|}
\hline $\begin{array}{c}\text { a) } \\
\text { PRINTEMPS } \\
\text { Séquences }\end{array}$ & $\begin{array}{c}\text { Nombre } \\
\text { d'an- } \\
\text { nées }\end{array}$ & Caractère \\
\hline $1481 / 1486$ & 6 & Variabilité \\
\hline $1487 / 1499$ & 13 & Chaud et sec \\
\hline $1500 / 1511$ & 12 & Variabilité \\
\hline $1512 / 1521$ & 10 & Humide et froid \\
\hline $1524 / 1537$ & 14 & Chaud et sec \\
\hline
\end{tabular}

\begin{tabular}{|c|c|c|}
\hline $\begin{array}{c}\text { b) ÉTÉ } \\
\text { Séquences }\end{array}$ & $\begin{array}{c}\text { Nombre } \\
\text { d'an- } \\
\text { nées }\end{array}$ & Caractère \\
\hline $1481 / 1490$ & 10 & Variabilité \\
\hline $1491 / 1504$ & 14 & Chaud et sec \\
\hline $1505 / 1508$ & 4 & Humide et froid \\
\hline $1508 / 1512$ & 5 & Chaud et sec \\
\hline $1513 / 1517$ & 5 & Humide et froid \\
\hline $1518 / 1524$ & 9 & Variabilité \\
\hline $1525 / 1534$ & 10 & Chaud et sec \\
\hline
\end{tabular}

Que ce soit pour le printemps ou l'été, on observe des séquences pluriannuelles, significatives par leur longueur, faisant alterner phases chaudes et sèches avec des séquences froides et humides. Dans l'économie rurale du début de la Renaissance, cette alternance entraîne des risques sérieux de disettes et est susceptible de gêner la croissance de plantes pérennes comme la vigne ou les arbres fruitiers qui, déjà, résistent mal aux grandes gelées de l'hiver.

Pour le blé et encore plus pour la vigne, la probabilité d'avoir un "bon " printemps suivi d'un "bon " été est fondamentale pour la qualité de la récolte (Tableau 9, infra). Quand on se penche sur les séquences jumelant le printemps et l'été, de façon à rendre compte des potentialités agronomiques, on note que les "bonnes années " sont relativement nombreuses, avec 28 occurrences de séquences " printemps + été " favorables à la croissance de la vigne et à la maturation du raisin (tableau 9), soit $40 \%$ des années. Mais les séquences "printemps + été " à dominante froide et humide sont présentes dans $20 \%$ des cas et les alternances "bon/mauvais " ou « mauvais/bon " sont au nombre de quatorze, soit une année sur quatre.

\section{La validation spatiale}

Il est difficile de valider dans l'espace les résultats de cette analyse, la chronique de Le Doyen étant la seule dans la France de l'Ouest à avoir été publiée pour le début de la Renaissance. Toutefois, on dispose pour le milieu de la période des deux livres de raison, de Versoris ${ }^{43}$ et du bourgeois de Paris ${ }^{44}$, qui, malgré des lacunes, donnent de précieuses indications pour la région parisienne. Comme chez Le Doyen, les remarques sont issues de leur vécu et relève donc du climat perçu.

43. Journal d'un bourgeois de Paris..., op. cit.

44. Livre de raison de Nicolas Versoris..., op. cit. 


\section{Tableau 9 - Fréquence des alternances climatiques des saisons printemps/été}

\begin{tabular}{|c|c|}
\hline Descriptif climatique des saisons printemps/été & Nombre de cas \\
\hline Chaud et sec/Chaud et sec & 22 \\
\hline Humide et froid/humide et froid & 11 \\
\hline Humide et froid/Chaud et sec & 5 \\
\hline Chaud et humide/Chaud et sec & 6 \\
\hline Chaud et sec/humide et froid & 4 \\
\hline Chaud et sec/chaud et humide & 1 \\
\hline Froid et sec/chaud et sec & 1 \\
\hline Froid et humide/chaud et humide & 1 \\
\hline Chaud et sec/froid et sec & 1 \\
\hline
\end{tabular}

Tableau 10 - Comparaisons entre les chroniques de Le Doyen, de Versoris et du "Bourgeois de Paris »

\begin{tabular}{|c|c|c|c|}
\hline & VERSORIS & BOURGEOIS DE PARIS & LE DOYEN \\
\hline 1517 & & $\begin{array}{l}\text { a) Vignes gelées dans tout } \\
\text { le Bassin parisien pendant } \\
5 \text { jours après Pâques } \\
\text { b) Grande sécheresse } \\
\text { autour de Paris avant le } \\
7 \text { juin } \\
\text { c) Après le } 7 \text { juin, pluies }\end{array}$ & $\begin{array}{c}\text { a) Gel de l'hiver jusqu'en } \\
\text { mai } \\
\text { Gel des vignes et des } \\
\text { arbres } \\
\text { b) On fauche jusqu'à la } \\
\text { Toussaint }\end{array}$ \\
\hline 1518 & & & Grands vents et pluies \\
\hline 1519 & & & Grand vent et grande pluie \\
\hline 1520 & $\begin{array}{l}17 \text { mars (ou16) : vent sur } \\
\text { tout le royaume }\end{array}$ & $\begin{array}{c}16 \text { mars (avant Pâques) : } \\
\text { tempête vers } 18 / 19 \mathrm{~h}\end{array}$ & $\begin{array}{l}\text { Pluie de printemps puis } \\
\text { été sec }\end{array}$ \\
\hline 1521 & Blés chers en avril et mai & & $\begin{array}{c}\text { Peu de grains. Importation } \\
\text { de grains }\end{array}$ \\
\hline 1522 & Pluie de printemps & & $\begin{array}{l}\text { Gel des vignes et genêts } \\
\text { Hiver très froid }\end{array}$ \\
\hline 1523 & $\begin{array}{l}\text { a) Pluie en juillet-août } \\
\text { b) Gelée à la Saint-Martin }\end{array}$ & $\begin{array}{l}\text { Gelée à la Saint-Martin } \\
\text { d'hiver qui dure } 6 \text { jours. } \\
\text { Gros dégâts qui se réper- } \\
\text { cutent jusqu'au printemps }\end{array}$ & $\begin{array}{l}\text { Gelée à la Saint-Martin } \\
\text { avec } 5 \text { jours de vent de } \\
\text { galerne. }\end{array}$ \\
\hline 1524 & $\begin{array}{l}\text { c) } 3 \text { mars : vent violent } \\
\text { a) Gelées l'hiver précédent } \\
\text { b) Printemps très } \\
\text { chaud avec sécheresse. } \\
\text { Procession en juin }\end{array}$ & & $\begin{array}{l}\text { Temps sec fin juin et tout } \\
\text { l'été }\end{array}$ \\
\hline 1525 & Année abondante en fruits & & Très bonne vendange \\
\hline 1526 & $\begin{array}{l}\text { a) Mi-février, crue de la } \\
\text { Seine } \\
\text { b) Récolte honnête }\end{array}$ & & Été bel et amoureux \\
\hline
\end{tabular}




\begin{tabular}{|c|c|c|c|}
\hline 1527 & $\begin{array}{c}\text { a) } 31 \text { mai : il pleut depuis } 6 \\
\text { semaines } \\
\text { Beau mois de juin } \\
\text { b) Peu de vin à cause } \\
\text { des froids de mars dernier }\end{array}$ & & $\begin{array}{c}\text { Hiver passable } \\
\text { Pluie au printemps jusqu'à } \\
\text { fin juillet } \\
\text { Peu de vin mais bon }\end{array}$ \\
\hline 1528 & $\begin{array}{c}\text { a) Jusqu'à fin mars, gelées. } \\
\text { Pluie, grêle... Crue de la } \\
\text { Seine. Gel des arbres et } \\
\text { des vignes } \\
\text { b) Fin mai : grand vent } \\
\text { et froid } \\
\text { Crue de la Seine } \\
\text { c) } 15 \text { juillet : orage, pluie, } \\
\text { grêle sur Île-de-France }\end{array}$ & $\begin{array}{c}27 \text { juillet : grêle sur } \\
\text { Chartres, } \\
\text { Picardie, Senlis, durant } 4 \\
\text { heures }\end{array}$ & $\begin{array}{l}\text { Grandes pluies } \\
\text { en avril et mai }\end{array}$ \\
\hline 1529 & $\begin{array}{l}\text { a) Début mars gelée sur } \\
\text { les vignes } \\
\text { b) Nuit du } 15 \text { avril : gelée } \\
\text { des vignes suite à une } \\
\text { pluie }\end{array}$ & & $\begin{array}{l}\text { Hiver avec grands vents } \\
\text { Pluie et neige }\end{array}$ \\
\hline 1530 & $\begin{array}{c}\text { c) } 29 \text { mars : vignes gelées } \\
\text { vers Orléans } \\
\text { d) } 3 \text { avril : grêle et très } \\
\text { froid, mais un brouillard } \\
\text { sauve les vignes. }\end{array}$ & $\begin{array}{c}\text { a) } 3 \text { semaines avant } \\
\text { Pâques, le } 17 \text { avril, jusqu'à } \\
\text { la Pentecôte, des proces- } \\
\text { sions contre le froid qui } \\
\text { gèle les vignes (gel tous } \\
\text { les matins) } \\
\text { Idem dans l'Orléanais. } \\
\text { b) Novembre à Tours; } \\
\text { récit des inondations du } \\
\text { Tibre à Rome, à Anvers, } \\
\text { Zélande, Rouen, Loire } \\
\text { d'Orléans, Seine à Paris. }\end{array}$ & $\begin{array}{l}\text { Hiver pluvieux avec crue } \\
\text { de la Mayenne }\end{array}$ \\
\hline 1531 & $\begin{array}{l}\text { a) } 19 \text { janvier : vent violent } \\
\text { b) dragon dans le ciel le } \\
\text { lendemain }\end{array}$ & $\begin{array}{c}\text { b) Le } 8 \text { janvier, grande } \\
\text { cornette au ciel entre } 9 \text { et } \\
\text { 10h. qui ressemble à un } \\
\text { dragon }\end{array}$ & $\begin{array}{c}\text { Hiver et printemps longs } \\
\text { et pluvieux } \\
\text { Bel été }\end{array}$ \\
\hline
\end{tabular}

Pour la plupart des années, les notations parisiennes correspondent au climat de Laval, en particulier pour les séquences pluvieuses dues probablement à des circulations d'ouest traversant la France. Mais quelques différences apparaissent, en particulier en 1530, où le froid semble circonscrit à l'est alors que l'Ouest est plus humide, ce qui laisserait penser à des séquences liées à de circulations méridiennes.

Emmanuel Le Roy Ladurie ${ }^{45}$ n'évoque pas l'ouest de la France, ne traitant que du nord, du centre et de l'est du royaume, avec des apports venant de Suisse et des Pays Bas à la suite des travaux de Van Engelen ${ }^{46}$ et de Pfister ${ }^{47}$. Parmi les années évoquées, 28 appartiennent à la séquence 1480-1537, celle de Le Doyen. Bien que les sources et les méthodes soient différentes, $71 \%$ d'entres elles fournissent des analyses proches, 7 \% sont

45. Le Roy LadURIE, Emmanuel, Histoire humaine..., op. cit.

46. VAn Engelen, A. F. V., Le Climat..., op. cit.

47. PfISTER, Christian, "Monthly temperature and precipitation in Central Europa from 1525 to 1800 ", dans : Bradley, Raymond et Jones, Philip, dir., Climate since 1500, London, Edit. Routlege, 1992, p. 118-142 
des nuances (1517-1536), alors que $22 \%$ donnent des résultats opposés (1485-1491-1496-1523-1530). Pour les printemps et les étés 1485, 1491, 1496 et 1530 , le climat de Laval apparaît plus chaud et moins humide que celui de l'Est alors que le contraire se produit en 1523.

Selon Michel Le Mené ${ }^{48}$, la première partie de la chronique a été écrite tardivement par Le Doyen et ceci pourrait expliquer la non concordance pour 1485, 1491 et 1496, sans qu'une hypothèse sur des circulations méridiennes dominantes puisse être éliminée. Les printemps et étés frais et humides des années 1535 à 1537 sont corroborées par des prix du blé élevés à Laval, alors que 1536, par exemple, a connu des vendanges tardives dans l'Est, ce qui renforce la probabilité de circulations méridiennes dominantes sur l'Europe. Ces différences montrent bien que le climat de Paris, a fortiori celui du nord et de l'est de la France, ne suffit pas à rendre compte du climat océanique de la France de l'Ouest et de ses nuances.

\section{Une rémission du Petit Âge Glaciaire (PAG)}

Malgré des hivers froids la variabilité interannuelle avec ses séquences de beau temps est marquée par des années favorables aux vendanges et laisse penser qu'une rémission du PAG a pu se produire vers 1500/1560, ce qu'E. Le Roy Ladurie appelle le " beau XVI ${ }^{\mathrm{e}}$ siècle ", au contraire du Xve siècle qui voit le début du PAG. Toutefois, faute de données sur la période qui précède, on est réduit pour Laval à des conjectures issues pour la plupart de constatations climatiques concernant la France du Nord et de l'Est. Au moins, pour ce qui est des hivers, la période 1481-1537 ne se marque pas à Laval par des périodes froides continues, bien que les années 1510-1524 aient connu une séquence de ce type et que l'hiver 1481 compte parmi les plus durs de l'Ancien Régime, comparable à ceux de 1709 ou 1740. La chronique commence par ces vers et ce n'est que l'une des deux années pour lesquelles Le Doyen débute sa description par le climat :

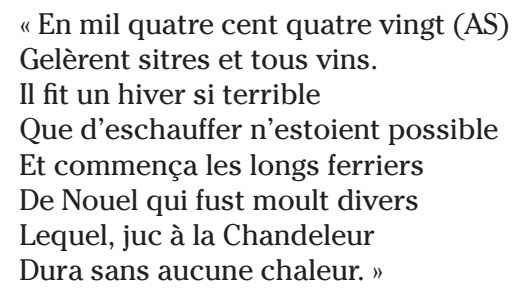

Le Doyen indique que les ceps de vigne ont, comme en 1481, gelé en 1511. Un certain nombre de ces hivers très froids se prolongent au-delà de Pâques (au moins 9 \% des années, soit une probabilité de retour décennale). Toutefois les hivers 1481, 1489, 1500, 1511 et 1512 «plus froids, plus âpres " que les autres représentent $9 \%$ de la série. On est proche du décile, même si ces hivers sont évoqués dans la première partie de la chronique.

48. LE MENÉ, Michel, Les campagnes angevines..., op. cit. 
A priori, si la variabilité interannuelle du climat est bien la marque d'un climat océanique atténué, les séquences de temps " correct " sont assez nombreuses pour que l'hypothèse de la rémission du PAG soit envisageable. Les périodes douces liées à des types de temps océanique dominent. Plus que pour l'hiver, les temps cléments du printemps et de l'été sont relativement nombreux et surtout se traduisent par des séquences significatives. On note certes des années " pourries " mais aussi des sécheresses (1527 à 1535 par exemple) avec des séquences qui pourraient rappeler celle de 1975-1976!

Faute de pouvoir connaître le climat lavallois avant 1481, on peut le comparer à celui du XVIII ${ }^{\mathrm{e}}$ siècle à partir de la chronique de Guittet de La Houllerie ${ }^{49}$, dont les indications, suffisamment précises, autorisent sur 20 ans (1756-1776) une reconstitution climatique mensuelle (tableau 11). Et, à la différence des informations de Le Doyen, les dates, les périodes sont définies climatiquement et non à interpréter en fonction des moissons et/ ou de la qualité du vin. Même si la période allant de 1756 à 1776 est plus tardive que celles des dernières années du règne de Louis XIV, les " années de misères " de Maurice Lachiver ${ }^{50}$, Guittet de La Houllerie a connu la fin du règne de Louis XIV, celui de Louis XV et le début de celui de Louis XVI. Il a donc vécu tous les épisodes les plus froids du PAG même s'il est vrai que les décennies 1720 et 1730 sont considérées comme chaudes ${ }^{51}$.

Tableau 11 - Fréquence de type de temps par mois selon Guitet de La Houllerie (1756-1776) (nombre d'épisodes sur la période, le cas échéant, plusieurs épisodes par mois)

\begin{tabular}{|c|c|c|c|c|c|c|c|c|c|c|c|c|c|}
\hline & janv. & fév. & mars & avril & mai & juin & juil. & août & sept. & oct. & nov. & déc. & total \\
\hline $\begin{array}{c}\text { Humide et } \\
\text { froid }\end{array}$ & 1 & 3 & 3 & 4 & 1 & & & 1 & & 1 & 1 & 1 & 16 \\
\hline Humide & 3 & 3 & 2 & & 2 & 2 & 4 & 4 & 2 & 2 & 3 & 3 & 30 \\
\hline $\begin{array}{c}\text { "Canicule" } \\
\text { froide }\end{array}$ & & & 2 & & 1 & 2 & & 1 & & & & 6 \\
\hline Froid et sec & 6 & 3 & 3 & 6 & 4 & 2 & 1 & & 1 & & 1 & 1 & 28 \\
\hline " Normal " & 10 & 11 & 14 & 9 & 13 & 10 & 9 & 12 & 16 & 16 & 16 & 14 & 150 \\
\hline Assez beau & 1 & & & 2 & 1 & 1 & 1 & 1 & & & & & 6 \\
\hline Grand beau & & & & 1 & 2 & 4 & 2 & 4 & 2 & & & & 15 \\
\hline
\end{tabular}

Guittet de La Houllerie fournit des descriptions plus précises concernant les grands froids et compare certains de ces hivers à ceux de 1709 et 1740 considérés comme les pires du XVIII ${ }^{\mathrm{e}}$ siècle.

49. Mémoire chronologique de Maucourt de Bourjolly..., op. cit.

50. LACHIVER, Marcel, Les années de misère. La famine au temps du Grand Roi, Paris, Fayard, 1991.

51. Le Roy LadurIE, Emmanuel, Histoire humaine..., op. cit. 
Le climat de Laval au début de la Renaissance

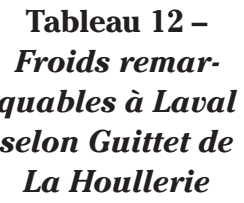

Tableau 12 -

Froids remarquables à Laval selon Guittet de

\begin{tabular}{|c|c|}
\hline & Années \\
\hline Mayenne gelée & $1757-1764-1766-1768-1776$ \\
\hline Gel & $1760-1767-1771-1772-1773$ \\
\hline Neige & 1771 \\
\hline Crues de la Mayenne & $1769-1772-1776$ \\
\hline
\end{tabular}

\begin{tabular}{|l|l|}
\hline 1766 & froid comme en 1740 \\
\hline 1768 & froid comme en 1709 \\
\hline 1776 & froid d'1 $1 / 4$ au dessous de 1740 et 1768 \\
\hline
\end{tabular}

De 1756 à 1776 on a un suivi uniquement climatique avec 74 occurrences de mauvais temps pour 35 de bon. Les mois sans commentaires (150 cas) doivent-ils être considérés comme " normaux ", c'est-à-dire n'ayant pas de caractère particulier à noter? Mais est-ce la même " normalité " que celle de Le Doyen, sachant que cette normalité est plus supposée par défaut, par une absence de données, que fondée sur des faits climatiques établis et que, de plus, cette supposée normalité est une hypothèse de la fin du $\mathrm{xx}$ siècle?

Si l'on compare les données de Le Doyen et de Guittet de La Houllerie, il semble bien que le deuxième quart du XVIII ${ }^{\mathrm{e}}$ siècle ait connu un climat plus rude et froid que celui du début du $\mathrm{XVI}^{\mathrm{e}}$, ne serait-ce que par la plus grande fréquence des grands hivers, et que la période 1481-1537 corresponde bien à une rémission du PAG dans le Bas Maine.

\section{La territorialisation du risque : les inondations}

Le Doyen nous indique 9 épisodes tout en signalant qu'en 1483, 1492 et 1508 , la Mayenne n'a pas débordé. Aucune autre source ${ }^{52}$ ne signale de crues de la Mayenne pour cette époque.

\section{Fréquences des crues}

Les crues d'hiver sont les plus nombreuses avec 5 occurrences mais les pluies de printemps entraînent également des débordements significatifs. Le Doyen nous signale donc 9 crues en 57 ans, soit une tous les 7 ans en moyenne. Faute de précisions sur leurs débordements et compte tenu du fait que la Mayenne n'était pas canalisée, on peut penser que ces inondations ont une fréquence au moins décennale. Mais pour trois d'entres elles, Le Doyen nous donne des précisions assez fines quant aux limites atteintes par les eaux.

52. Champion, Maurice, Les inondations en France du $X^{e}$ siècle à nos jours, Paris, Dalmont et Dunod, 6 volumes, 1860 (réédition CEMAGREF, 2001); Musset, René, Le Bas Maine..., op. cit. 


\section{Tableau 14 - Épisodes de crues indiqués par G. Le Doyen entre 1481 et 1537}

\begin{tabular}{|c|c|c|c|c|}
\hline Années & Saison & Extension & Genèse & Retour \\
\hline 1481 & Printemps & $\begin{array}{l}\text { " Ruisseau furent moult grands " } \\
\text { jusqu'à Saint-Vénérand. } \\
\text { Rue du pont de Mayenne sous l'eau }\end{array}$ & Crue de débâcle & Trentennale \\
\hline 1494 & Hiver & $\begin{array}{l}\text { " Hiver assez pesant " et les rivières } \\
\text { furent hors des rives " }\end{array}$ & $\begin{array}{l}\text { Sols saturés et climat } \\
\text { humide. Neige (?) }\end{array}$ & Décennale \\
\hline 1497 & Hiver & $\begin{array}{l}\text { "Les rivières furent moult grandes } \\
\text { car il rompit plusieurs étangs " }\end{array}$ & & Décennale \\
\hline 1502 & Hiver & $\begin{array}{l}\text { "Les eaux furent grandes [...] qui } \\
\text { tinrent longues et ennuyeuses qu'ils } \\
\text { surmontaient et chaussées et mai- } \\
\text { sons, qu'après faillit en besogner } \\
\text { maçons" }\end{array}$ & $\begin{array}{l}\text { Hiver doux et plu- } \\
\text { vieux }\end{array}$ & Trentennale \\
\hline 1509 & Printemps & $\begin{array}{l}\text { "Eaux hors de leurs rivages mais ne } \\
\text { firent pas grands dommages" }\end{array}$ & Mai pluvieux & Décennale \\
\hline $\begin{array}{l}1510 \\
1511\end{array}$ & $\begin{array}{l}\text { Automne } \\
\text { Hiver }\end{array}$ & $\begin{array}{c}\text { "La rivière gela [...] que glaçons } \\
\text { moult pervers firent moult grands } \\
\text { dommages par où ils faisaient leurs } \\
\text { passages ". }\end{array}$ & Crue de débâcle & \\
\hline 1511 & Automne & $\begin{array}{c}\text { "Les grands desrays des eaux ont } \\
\text { été partout au temps de toussaint } \\
\text { et devant qu'il n'est ruisseau qui ne } \\
\text { fût grand " }\end{array}$ & $\begin{array}{l}\text { "La pluie n'avait nulle } \\
\text { cesse jour et nuit. } \\
\text { Semaison ne dut être } \\
\text { faite" }\end{array}$ & Décennale \\
\hline 1530 & Automne & $\begin{array}{c}\text { Faubourg Saint-Vénérand et hôpital } \\
\text { Saint-Julien inondés } \\
\text { «Elle fut près de Saint-Vénérand. } \\
\text { Jusqu'au devant du grand four de } \\
\text { Saint-Julien... Le carrefour de la } \\
\text { Sereine était tombé en grandes } \\
\text { ruines. " }\end{array}$ & $\begin{array}{l}\text { Pluie et fontes de } \\
\text { neiges sur sol saturé } \\
\text { "Hiver courtois et } \\
\text { doux [...] moult } \\
\text { pluvioux... Par neige } \\
\text { pluie ou vent ". }\end{array}$ & $\begin{array}{l}\text { Trentennale } \\
\text { et peut être } \\
\text { plus } \\
\text { "Car de vie } \\
\text { d'homme } \\
\text { ne fust si } \\
\text { grand ni sui } \\
\text { austère". }\end{array}$ \\
\hline 1535 & Printemps & "Ruisseau étaient grandes rivières " & $\begin{array}{l}\text { "Jusqu'à la Madeleine } \\
\text { pleuvait sans cesse " }\end{array}$ & Décennale \\
\hline
\end{tabular}

La genèse et l'estimation des retours sont des déductions des auteurs en l'absence de précisions de Le Doyen (notes de Le Doyen en italique).

La carte la plus ancienne de la ville date de $1753^{53}$, soit plus de 200 ans après la fin de la chronique. Mais ces prairies de la rive droite ont été protégées de l'urbanisation pour le blanchiment des toiles qui fut la richesse de la ville pendant toute l'époque moderne. Frédérique Pitou note que la pression des tisserands et des filateurs des toiles de Laval a " figé l'espace urbain au centre de la ville ${ }^{54}$ ". La carte de 1753 (figure 3) montre bien l'urbanisation sur les premières pentes de la rive droite, le faubourg de Saint-Vénérand et les prairies sur lesquelles sont blanchies les toiles. Le côté "zone humide " de ces prairies est par ailleurs signalé par Le Doyen

53. COUANIER DE LAunay, S. L., Histoire de Laval, Laval, Godbert éditeur, 1856. [http://books. google.fr/books?id=I3C7wH_S9LIC\&printsec=frontcover $\# v=$ onepage \& $q \& f=$ false $]$.

54. PITOU, Frédérique, Laval au XVIII siècle, Marchands, artisans, ouvriers dans une ville textile, Laval, La Mayenne : archéologie, histoire, supplément n 8, 1996. 
qui écrit que, pour construire le nouvel hôpital Saint-Julien, il a fallu le faire sur pilotis (1528).

On peut faire l'hypothèse que, tout en servant de réserves foncières pour le blanchiment des toiles, ces prairies étaient également, en l'absence de digues, des zones inondables. Elles devaient être submergées lors des crues mineures, au moins par les crues décennales. Par contre, quand Le Doyen localise les maisons touchées lors de crues noyant Saint-Vénérand, on retrouve une fréquence de deux à trois épisodes sur près de soixante ans. C'est le cas pour les trois crues hivernales de 1481, 1502 et 1530 (figure 3). Deux d'entre elles se produisent à la fin d'hivers particulièrement froids. Même si on est aux alentours de 1500 en phase de rémission du petit âge glaciaire, les crues de débâcle et de fonte des neiges sont dominantes et donnent les inondations les plus importantes, même si ces débits de crues ne sont pas connus.

Figure 3 - Crues décennales et trentennales et lieux inondés signalés par G. Le Doyen en 1530 Le fond de carte est repris de la carte de Laval en 1753

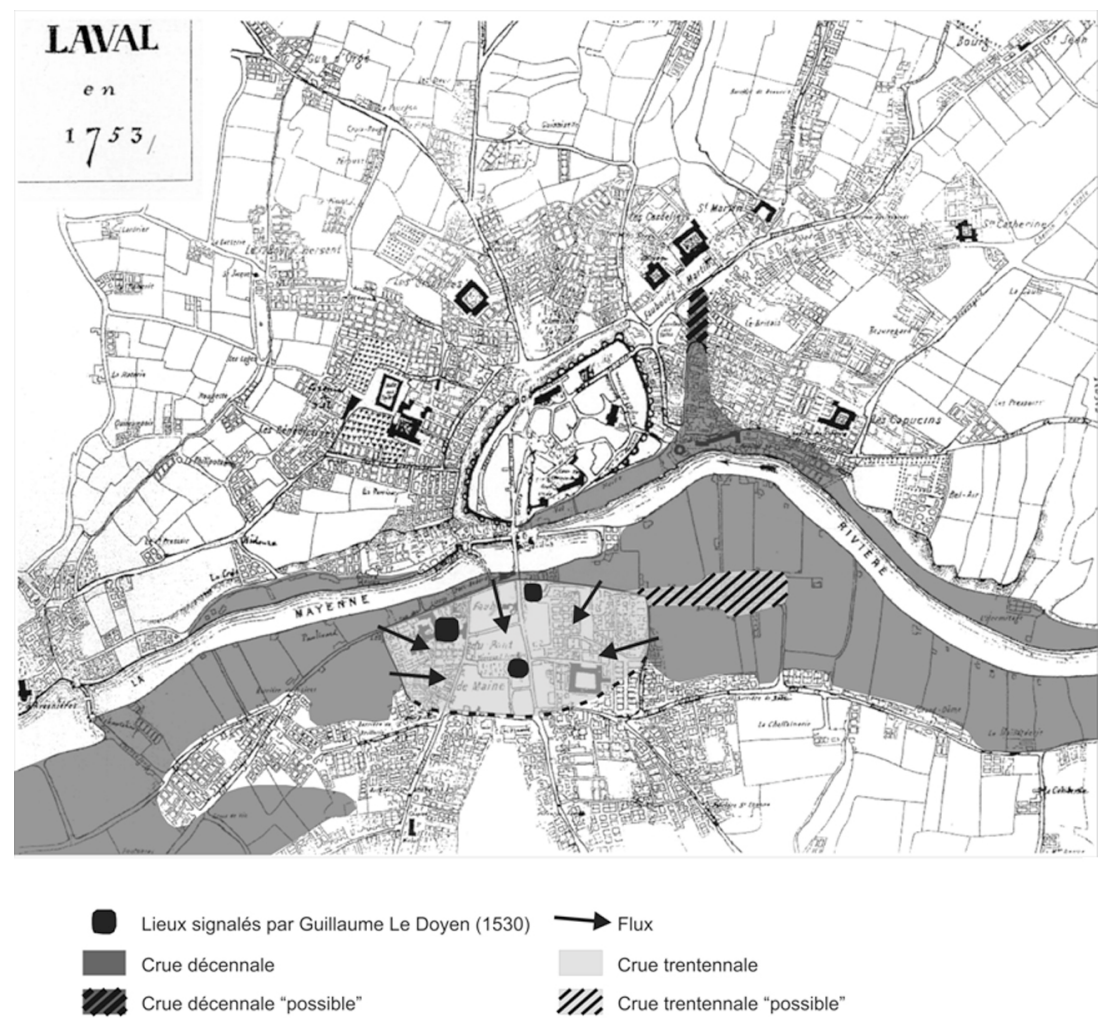




\section{Territoires du risque, climat et carte mentale}

Alors qu'en est-il des territoires du risque vécu par Le Doyen? On peut, comme on vient de le voir pour les inondations, proposer, en suivant les écrits de Le Doyen, une cartographie de ce qu'ont vécu les habitants de Laval de 1480 à 1537. En écrivant sa chronique, il nous donne une idée de ce que représentait pour lui et pour une catégorie sociale instruite et aisée, ce que l'on nommerait actuellement des risques d'origine climatique. On peut ainsi dessiner une carte mentale (figure 4) des espaces à risques de Guillaume Le Doyen, pour lesquels le climat joue peu ou prou un rôle significatif.

On a choisi ici une modélisation graphique qui tient compte des distances entre le plus proche - le quartier de Saint-Vénérand - et les territoires lointains comme l'Orléanais, fournisseur de bon vin, ou sujets à des inondations dont la connaissance était parvenue jusqu'à Laval. L'échelle choisie s'inspire d'une distance logarithmique qui met l'accent sur les territoires proches. Les figurés vont du plus proche, les lieux inondés dans le quartier Saint-Vénérand, aux inondations de Saumur, Orléans. Il s'agit là d'aléas dont la cause directe est le climat. Mais les environs de Laval, dans ce qui constitue le territoire habituel parcouru par Le Doyen et qui le ravitaille en vin, blé et bois, sont pour lui sources d'inquiétude dès que le climat se détériore et menace de troubler son confort. C'est surtout vrai pour le bois et le vin, moins pour le blé, car il compatit sur ce point aux malheurs de ses concitoyens. On peut ainsi cartographier les espaces de production des produits à risques et les solutions apportées, par exemple les importations de blé et de vin.

L'objectif de cette recherche était de reconstituer la variabilité climatique sur une longue période en l'absence de données chiffrées et sans avoir les référents classiques que sont les glaciers, le gel des canaux et les dates de vendanges, en n'utilisant donc que des données textuelles et qualitatives. La recherche, pilotée par de grandes séquences de types de temps " perçus " par leur durée et leur intensité, montre l'importance du climat vécu dont l'étude correspond à l'un de trois objectifs fixés à la place de la géographie dans l'étude du climat ${ }^{55}$.

La reconstitution de ce climat vécu devient ainsi une aide à la détermination des climats passés et va au-delà d'une géographie des représentations. Les données relatives aux vendanges et à la qualité des moissons ainsi que l'approche de l'aléa hivernal ont permis de proposer un calendrier des types de temps saisonniers probables et d'appréhender la variabilité interannuelle en allant au-delà de la simple alternance de séquences annuelles plus ou moins favorables aux activités économiques. Pour cela, l'échelle saisonnière a semblé la plus synthétique, sous réserve d'estimer l'indice de confiance que l'on accorde aux conclusions issues d'une source unique.

55. PÉGuY, Charles Pierre et MARChAND, Jean-Pierre, " Climatologie et culture ", op. cit. 
Le climat de Laval au début de la Renaissance

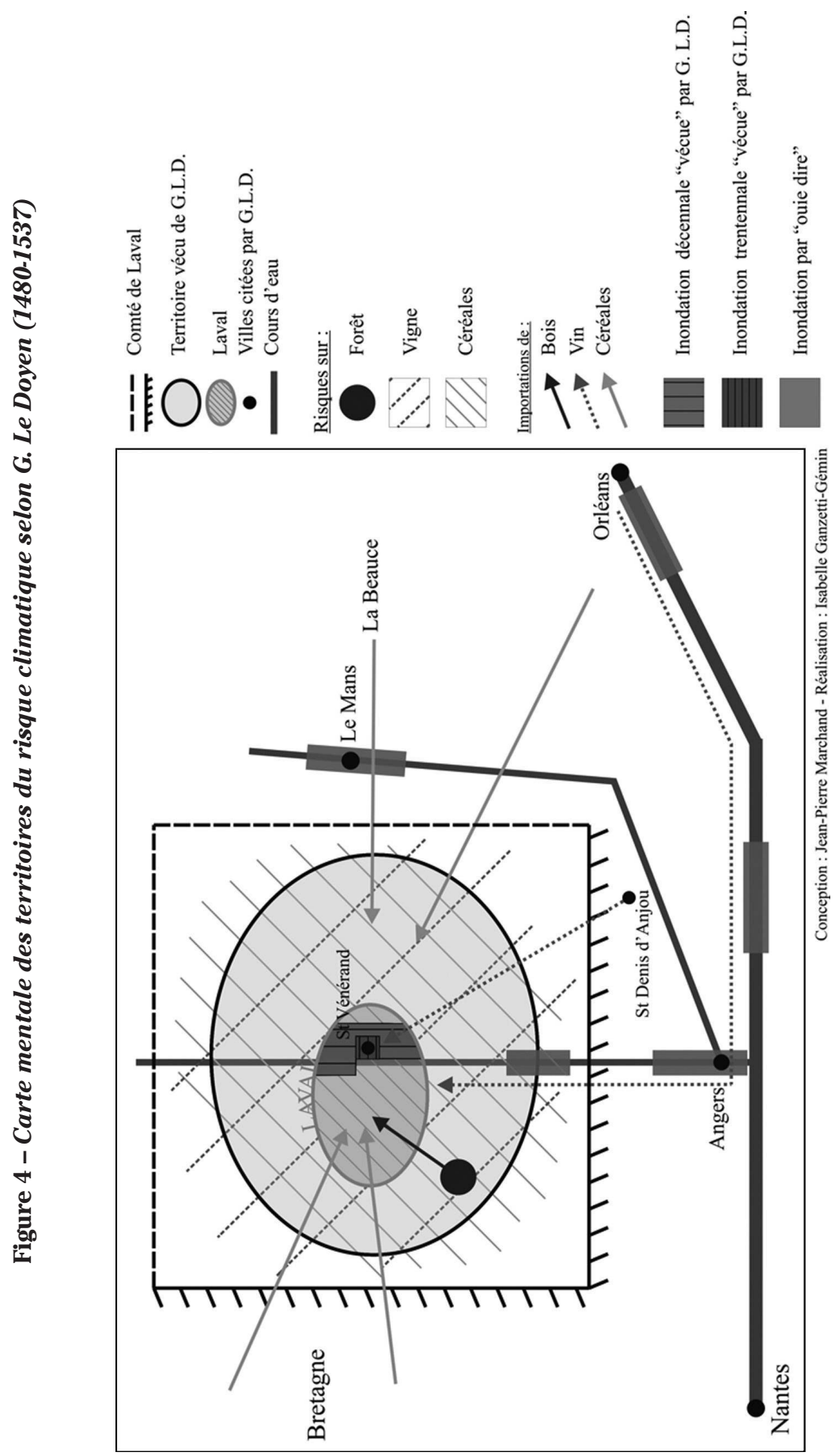


Cette reconstitution a permis de valider l'hypothèse d'une rémission du PAG dans l'ouest de la France, tout en montrant que pour certaines années des différences notables peuvent apparaître avec des régions plus orientales. Quant à savoir si la "station " de Laval est représentative du climat de l'Ouest, c'est un autre problème qui suppose la constitution et l'exploitation d'une base de données sur la Bretagne, le Maine et l'Anjou. Enfin la description des crues fournie par le Doyen a permis d'en déterminer la fréquence et de cartographier les zones inondables.

Les Annales et chronique du païs de Laval n'en sont que plus précieuses pour appréhender le climat océanique à la fin du $\mathrm{XV}^{\mathrm{e}}$ et au début du $\mathrm{XVI}^{\mathrm{e}}$ siècle. À Laval, au tournant $\mathrm{du} \mathrm{XVI}^{\mathrm{e}}$ siècle, seul le climat vécu peut être appréhendé, mais il aide à approcher le gisement climatique et permet, via l'étude des crues, de voir les modifications territoriales apportées par les interactions entre le climat et la société.

\section{Remerciements}

Les auteurs remercient vivement Brigitte Maillard, professeur émérite d'histoire moderne à l'université de Tours et Daniel Pichot, professeur émérite d'histoire médiévale à l'université de Rennes 2 pour leurs relectures attentives du texte et des notes infrapaginales. 


\section{RÉSUMÉ}

L'enquête sur le climat à la fin du Moyen Âge, pendant ce que les historiens appellent le " petit âge glaciaire " (PAG) est demeurée jusqu'ici presqu'inexistante pour l'Ouest de la France par manque de données exploitables. Le présent article essaie de combler ce déficit en utilisant la " Chronique " du notaire lavallois Guillaume Le Doyen. Ce texte en vers de piètre qualité relate les événements locaux entre 1481 et 1537 et, comme souvent à l'époque, il retient quantité de notations météorologiques qui peuvent permettre d'étudier le climat de l'Ouest pendant presque 60 ans. Une fois établi le degré de fiabilité des notations et en considérant que Le Doyen nous livre avant tout le climat vécu, l'étude devient possible. L'hiver est bien repéré comme le printemps et l'été. L'étude de la variabilité aboutit à constater à cette époque une rémission dans le petit âge glaciaire, les périodes froides n'étant pas dominantes. L'étude se termine par l'examen des inondations à Laval auxquelles l'auteur est évidemment sensible. La chronique permet une approche du climat tempéré de l'Ouest, sensiblement nuancé par rapport à ce qui est observé à Paris, mais il faudrait pouvoir étendre l'enquête à d'autres sources pour confirmer cela.

\section{ABSTRACT}

Very little research into the climate of the late Middle Ages, during what historians call the "little ice age", has hitherto been undertaken for western France because of the paucity of data. This article seeks to address this issue by using the chronicle of the Laval notary Guillaume Le Doyen. This text in poor quality verse relates local events between 1481 and 1537 and, as was often the case in this period, it contains much meteorological information that can be used to study the climate of western France over almost sixty years. This analysis can been undertaken once the trustworthiness of the information has been established and once provision is made for the fact that Le Doyen described the climate as he perceived it. Data is available for spring, summer and winter and allows us to conclude that the little ice age was in remission; the cold periods were no longer dominant. The study ends with an overview of floods of which the author was particularly aware. The chronicle allows a new analysis of the temperate climate of western France, with notable differences from what has been observed in Paris, though it would be necessary to expand the survey to other sources to confirm this impression. 